Pacific

Journal of

Mathematics

\title{
GAMMA FACTORS OF DISTINGUISHED REPRESENTATIONS OF $\mathrm{GL}_{\boldsymbol{n}}(\mathbb{C})$
}

\section{AlEXANDER KEMARSKY}




\title{
GAMMA FACTORS OF DISTINGUISHED REPRESENTATIONS OF GL $\mathbf{G}_{n}(\mathbb{C})$
}

\author{
ALEXANDER KEMARSKY
}

Let $(\pi, V)$ be a $\mathbf{G L}_{n}(\mathbb{R})$-distinguished, irreducible, admissible representation of $G_{n}(\mathbb{C})$, let $\pi^{\prime}$ be an irreducible, admissible, $G_{L}(\mathbb{R})$-distinguished representation of $\mathrm{GL}_{m}(\mathbb{C})$, and let $\psi$ be a nontrivial character of $\mathbb{C}$ which is trivial on $\mathbb{R}$. We prove that the Rankin-Selberg gamma factor at $s=1 / 2$ is $\gamma\left(1 / 2, \pi \times \pi^{\prime} ; \psi\right)=1$. The result follows as a simple consequence from the characterization of $G_{n}(\mathbb{R})$-distinguished representations in terms of their Langlands data.

1. Introduction

2. Notation and preliminaries

3. Some matrix spaces decompositions

142

4. Proof of Theorem 1.2

5. Calculation of Rankin-Selberg gamma factors

149

6. Integral representation of Whittaker functions

154

7. Archimedean Asai integrals

160

8. Equality of two functionals

162

Appendix A. Generic Langlands quotient 165

Appendix B. Gamma factors: converse direction 166

Acknowledgements 170

$\begin{array}{ll}\text { References } & 170\end{array}$

\section{Introduction}

Let $G_{n}(\mathbb{C})=\mathrm{GL}_{n}(\mathbb{C}), G_{n}(\mathbb{R})=\mathrm{GL}_{n}(\mathbb{R})$. Let $B_{n}=B_{n}(\mathbb{C})$ be the Borel subgroup of upper triangular matrices in $G_{n}(\mathbb{C})$. Denote the complex conjugation by $x \rightarrow \bar{x}$. We identify $G_{n}(\mathbb{C}) / G_{n}(\mathbb{R})$ with the space of matrices

$$
X_{n}=\left\{x \in G_{n}(\mathbb{C}): x \cdot \bar{x}=I_{n}\right\},
$$

This research was supported by ISF grant No. 1394/12.

MSC2010: primary 22E30; secondary 20G05, 11F70.

Keywords: Rankin-Selberg gamma factor, distinguished representations, Langlands classification,

Whittaker model. 
via the isomorphism $g G_{n}(\mathbb{R}) \mapsto g \cdot \bar{g}^{-1}$. See [Serre 2002, Chapter 3, Section 1 , Lemma 1] for the proof of the surjectivity of this map. Given a representation $\pi$ of $G_{n}(\mathbb{C})$, the representation $\bar{\pi}$ is defined by the formula $\bar{\pi}(g):=\pi(\bar{g})$.

The group $G_{n}(\mathbb{C})$ acts on $X_{n}$ by the twisted conjugation, where the action is induced by the natural action $l(g) g^{\prime} G_{n}(\mathbb{R}):=g g^{\prime} G_{n}(\mathbb{R})$. Namely, we have

$$
g^{\prime} G_{n}(\mathbb{R}) \leftrightarrow g^{\prime} \cdot{\overline{g^{\prime}}}^{-1}:=x \quad \text { and } \quad l(g)\left(g^{\prime} G_{n}(\mathbb{R})\right):=g g^{\prime} G_{n}(\mathbb{R}) \leftrightarrow g g^{\prime} \bar{g}^{\prime}-1 \bar{g}^{-1} .
$$

Hence, the action of $G_{n}(\mathbb{C})$ on $X$ is given by $l(g) x:=g x \bar{g}^{-1}$.

For a topological vector space $V$, we denote by $V^{*}$ the topological dual of $V$, i.e., the space of all continuous maps from $V$ to $\mathbb{C}$. In this paper we work with the category of the admissible smooth Fréchet representations of moderate growth (see [Wallach 1992, Section 11.5; Aizenbud et al. 2008, Section 2.1]).

A representation $(\pi, V)$ of $G_{n}(\mathbb{C})$ is called $G_{n}(\mathbb{R})$-distinguished if there exists a nonzero continuous linear map $L: V \rightarrow \mathbb{C}$ such that

$$
L(\pi(h) v)=L(v) \quad \text { for all } v \in V, h \in G_{n}(\mathbb{R}) .
$$

We denote the space of all such linear maps by $\left(V^{*}\right)^{G_{n}(\mathbb{R})}$. We denote the set of equivalence classes of irreducible representations of $G_{n}(\mathbb{C})$ by $\operatorname{Irr}\left(G_{n}(\mathbb{C})\right)$ and the set of equivalence classes of irreducible $G_{n}(\mathbb{R})$-distinguished representations of $G_{n}(\mathbb{C})$ by $\operatorname{Irr}_{G_{n}(\mathbb{R})}\left(G_{n}(\mathbb{C})\right)$.

Let $\psi: \mathbb{C} \rightarrow \mathbb{C}^{\times}$be a nontrivial unitary character which is trivial on $\mathbb{R}$, for example

$$
\psi(x)=e^{\pi(x-\bar{x})} .
$$

We let $U_{n}(\mathbb{C})$ be the group of upper triangular matrices with unit diagonal and we denote by $\theta_{\psi, n}$ the character $\theta_{\psi, n}: U_{n}(\mathbb{C}) \rightarrow \mathbb{C}^{\times}$defined by

$$
\theta_{\psi, n}(u)=\psi\left(\sum_{i=1}^{n-1} u_{i, i+1}\right) .
$$

A $\psi$-form on $V$ is a nonzero continuous linear form $\lambda: V \rightarrow \mathbb{C}$ such that

$$
\lambda(\pi(u) v)=\theta_{\psi, n}(u) v,
$$

for each $v \in V$ and each $u \in U_{n}(\mathbb{C})$. We say that $\pi$ is a generic representation if there exists a $\psi$-form on $V$.

Theorem 1.1. Let $\pi \in \operatorname{Irr}_{G_{t}(\mathbb{R})}\left(G_{t}(\mathbb{C})\right)$ and let $\pi^{\prime} \in \operatorname{Irr}_{G_{r}(\mathbb{R})}\left(G_{r}(\mathbb{C})\right)$. If $\psi$ is a nontrivial character of $\mathbb{C}$ with a trivial restriction to $\mathbb{R}$ then the value of the RankinSelberg gamma factor at $s=1 / 2$ is

$$
\gamma\left(\frac{1}{2}, \pi \times \pi^{\prime} ; \psi\right)=1
$$


A similar theorem is proved in [Offen 2011, Theorem 0.1] for the $p$-adic case (see also [Ok 1997]). See Section 5 for the definition of Rankin-Selberg integrals, Rankin-Selberg gamma factors and for the proof of Theorem 1.1.

We will deduce Theorem 1.1 from the characterization of irreducible $G_{n}(\mathbb{R})$ distinguished representations of $G_{n}(\mathbb{C})$. Let $\chi$ be a character of $B_{n}$. We denote by $I(\chi)$ the normalized parabolic induction representation

$$
I(\chi):=\operatorname{Ind}_{B_{n}}^{G_{n}(\mathbb{C})}(\chi)
$$

of the character $\chi=\left(\chi_{1}, \ldots, \chi_{n}\right)$ from $B_{n}$ to $G_{n}(\mathbb{C})$. We remind the reader that this space consists of smooth functions such that $f(b g)=\left(\chi \delta^{1 / 2}\right)(b) f(g)$ for all $b \in B_{n}$ and all $g \in G_{n}(\mathbb{C})$. The group $G_{n}(\mathbb{C})$ acts on $I(\chi)$ by right translations, and the group of permutations on $n$ elements, $S_{n}$, acts naturally on the characters of $B_{n}$. We will call a character $\chi=\left(\chi_{1}, \chi_{2}, \ldots, \chi_{n}\right)$ of $B_{n}$ dominant if

$$
|\chi(t)|=\left|t_{1}\right|^{\lambda_{1}}\left|t_{2}\right|^{\lambda_{2}} \cdots\left|t_{n}\right|^{\lambda_{n}} \quad \text { with } \lambda_{1} \geq \lambda_{2} \geq \cdots \geq \lambda_{n} .
$$

In Section 4 we will prove the following.

Theorem 1.2. Let $\pi$ be an element of $\operatorname{Irr}_{G_{n}(\mathbb{R})}\left(G_{n}(\mathbb{C})\right)$ and let $\chi=\left(\chi_{1}, \chi_{2}, \ldots, \chi_{n}\right)$ be a dominant character of $B_{n}$. Suppose $\pi$ is the Langlands quotient of $I(\chi)$, that is, the unique irreducible quotient of $I(\chi)$. Then there exists an involution $w \in S_{n}$ such that $w \chi=\left(\overline{\chi^{-1}}\right)$. Moreover, we can choose this $w$ such that for every fixed point $i$ of $w$ we have $\chi_{i}(-1)=1$.

Remark 1.3. Note that the conditions $w(i)=i, \chi_{w(i)}=\bar{\chi}_{i}^{-1}$ and $\chi_{i}(-1)=1$ imply that $\chi_{i}$ is $\mathrm{GL}_{1}(\mathbb{R})$-distinguished. Indeed, $\chi_{i}=\bar{\chi}_{i}^{-1}$ implies that $\chi_{i}$ is $\mathbb{R}_{+}$-invariant. Together with the condition $\chi_{i}(-1)=1$ this means that $\chi_{i}$ is $\mathbb{R}^{\times}$-invariant (i.e., $\mathrm{GL}_{1}(\mathbb{R})$-invariant).

As a consequence of Theorem 1.2 we obtain the following analogue of [Aizenbud and Lapid 2012, Theorem B.1].

Theorem 1.4. Let $\pi \in \operatorname{Irr}_{G_{n}(\mathbb{R})}\left(G_{n}(\mathbb{C})\right)$ and suppose $\pi$ is a generic representation of $G_{n}(\mathbb{C})$. Then $\bar{\pi} \simeq \tilde{\pi}$, where $\tilde{\pi}$ is the contragredient representation of $\pi$.

Let $(\pi, V)$ be an irreducible representation of $G_{n}(\mathbb{C})$. The existence of $I(\chi)$ with the properties stated in Theorem 1.2 is a well-known fact (see [Wallach 1988, Theorem 5.4.1]). It is also well-known that the Langlands quotient of $I(\chi)$ is generic if and only if $I(\chi)$ is irreducible (see Appendix A). Therefore, $\bar{\pi}=I(\bar{\chi})$ and $\tilde{\pi}=I\left(\chi^{-1}\right)$. Since $\bar{\pi}$ is irreducible, for every $w_{0} \in S_{n}$ we have $I\left(w_{0}(\bar{\chi})\right) \simeq I(\bar{\chi})$. In particular, for $w \in S_{n}$ such that $w(\bar{\chi})=\chi^{-1}$, we have

$$
\bar{\pi} \simeq I(\bar{\chi}) \simeq I(w(\bar{\chi})) \simeq I\left(\chi^{-1}\right) \simeq \tilde{\pi}
$$


A similar result was proved by Marie-Noelle Panichi in her Ph.D. thesis [2001, Theorem 3.3.6].

The structure of the paper is as follows. In Section 3 we recall basic facts about the structure of $G_{n}(\mathbb{C})$. In Section 4 we prove Theorem 1.2 by analyzing the geometry of the action of $B_{n}$ on the variety $G_{n}(\mathbb{C}) / G_{n}(\mathbb{R})$. In Section 5 , as an application of our classification we deduce Theorem 1.1. In Section 8 we prove a new type of integral identity for Whittaker functions on generic $G_{n}(\mathbb{R})$-distinguished representations which in turn proves [Lapid and Mao 2014, Assumption 5.2]. A similar identity was proved in the $p$-adic case in [Offen 2011, Corollary 7.2]. Our proof is similar to the proof in the $p$-adic case, but in the archimedean case there are many analytical difficulties. We overcome them in Sections 5-7.

Finally, in Appendix B we prove a converse-type theorem. We prove that if $(\pi, V)=I(\chi)$ is an irreducible, generic, admissible unitary representation of $G_{n}(\mathbb{C})$ such that for every unitary character $\chi^{\prime}(z)=(z /|z|)^{2 m}$ with $m \in \mathbb{Z}$ we have

$$
\gamma\left(\frac{1}{2}, \pi \times \chi^{\prime}, \psi\right)=1
$$

then $\pi$ is $G_{n}(\mathbb{R})$-distinguished. The proof is done by a combinatorial argument combined with the Tadic-Vogan classification of the unitary dual of $G_{n}(\mathbb{C})$.

\section{Notation and preliminaries}

Let $M(a \times b, F)$ be the space of matrices with $a$ rows and $b$ columns with entries in $F$, where $F$ is either $\mathbb{R}$ or $\mathbb{C}$. Let $\eta_{n}=(0,0, \ldots, 1)$ be an element of $M(1 \times n, \mathbb{R})$, and let $P_{n}(\mathbb{R})$ be the subgroup of $G_{n}(\mathbb{R})$ consisting of all $n \times n$ matrices with the last row equal to $\eta_{n}$.

Let $U_{n}(F)$ be the group of all upper triangular matrices in $M(n \times n, F)$ with unit diagonal. Let

$$
K_{n}=\left\{g \in G_{n}(\mathbb{C}): g \cdot{ }^{t} \bar{g}=I\right\}
$$

be the standard maximal compact subgroup of $G_{n}(\mathbb{C})$.

For $g \in G_{n}(\mathbb{C})$, define

$$
\|g\|:=\sqrt{\sum_{i, j=1}^{n}\left|g_{i j}\right|^{2}} \text { and }\|g\|_{H}:=\max \left(\|g\|,\|g\|^{-1}\right) .
$$

The value $\|g\|_{H}$ is called a norm on $g$ (see [Wallach 1988, Section 2.A.2] for a general discussion on norms on a reductive group).

Let $G$ be a group and $H$ its subgroup. We say that a function $f: G \rightarrow \mathbb{C}$ is $H$-finite if the dimension of the space spanned by right $H$-translations of $f$ is finite. In this work we will often consider $K_{n}$-finite functions on $G_{n}(\mathbb{C})$. 
For $V$, a finite dimensional vector space over $\mathbb{R}$, we denote by $\mathcal{S}(V)$ the Schwartz space of all infinitely differentiable functions $f: V \rightarrow \mathbb{C}$ of rapid decay.

Let $\Phi \in \mathcal{S}(V)$, where $V=M(a \times b, \mathbb{C})$. We denote by $\hat{\Phi}$ the Fourier transform of $\Phi$. It is a function on the same space, defined by

$$
\hat{\Phi}(X)=\int \Phi(Y) \psi\left(-\operatorname{Tr}\left({ }^{t} X Y\right)\right) d Y .
$$

For $\Phi \in \mathcal{S}\left(\mathbb{C}^{n}\right)$ and $g \in G_{n}(\mathbb{C})$ we denote by $(R(g) \Phi)(x):=\Phi(x g)$ the right translation of $\Phi$ by $g$.

For $z=x+i y \in \mathbb{C}$ we denote by $|z|=\sqrt{x^{2}+y^{2}}$ the usual absolute value of $z$ and by $|z|_{\mathbb{C}}=|z|^{2}=x^{2}+y^{2}$ the square of the usual absolute value. Note that $\mu(z A)=|z|_{\mathbb{C}} \mu(A)$, where $A \subset \mathbb{C}$ is an open set and $\mu$ is a Haar measure on $\mathbb{C}$.

Let $W_{n}$ equal $S_{n}$ and let $W_{n, 2}=\left\{w \in W_{n}: w^{2}=1\right\}$ be the set of involutions in $W_{n}$. For $w \in W_{n, 2}$ set

$$
I_{w}=\{(i, j): i>j, w(i)>w(j)\},
$$

and define for any function $\kappa: I_{w} \rightarrow \mathbb{Z}_{\geq 0}$ a character $\alpha_{\kappa}$ of $B_{n}$ by the formula

$$
\alpha_{\kappa}\left(\operatorname{diag}\left(t_{1}, \ldots, t_{n}\right)\right)=\prod_{(i, j) \in I_{w}}\left[\frac{t_{i}}{t_{j}}\right]^{\kappa(i, j)} .
$$

We will identify $\alpha_{\kappa}$ with the one-dimensional representation of $B_{n}$ on the vector space $\mathbb{C}$ with the action of $\alpha_{\kappa}$. By abuse of notation we will denote both the function and the one-dimensional representation by the same symbol, $\alpha_{\kappa}$.

For the convenience of the reader we write here notation and formulations of some of the theorems that appear in [Aizenbud and Lapid 2012], in versions that are suitable for this work.

Let $G$ be an arbitrary group.

- For any $G$-set $X$ and a point $x \in X$, we denote by $G(x)$ the $G$-orbit of $x$ and by $G^{x}$ the stabilizer of $x$.

- For any representation of $G$ on a vector space $V$ and a character $\chi$ of $G$, we denote by $V^{G, \chi}$ the subspace of $(G, \chi)$-equivariant vectors in $V$.

- Given manifolds $L \subseteq M$, we denote by $N_{L}^{M}:=\left(T_{M \mid L}\right) / T_{L}$ the normal bundle to $L$ in $M$ and by $C N_{L}^{M}:=\left(N_{L}^{M}\right)^{*}$ the conormal bundle. For any point $y \in L$, we denote by $N_{L, y}^{M}$ the normal space to $L$ in $M$ at the point $y$ and by $C N_{L, y}^{M}$ the conormal space to $L$ in $M$ at the point $y$.

- The symmetric algebra of a vector space $V$ is denoted by

$$
\operatorname{Sym}(V)=\bigoplus_{k \geq 0} \operatorname{Sym}^{k}(V) .
$$


- The Fréchet space of Schwartz functions on a Nash manifold $X$ is denoted by $\mathcal{S}(X)$ and the dual space of Schwartz distributions by $\mathcal{S}^{*}(X):=\mathcal{S}(X)^{*}$.

- For any Nash vector bundle $E$ over $X$ we denote by $\mathcal{S}(X, E)$ the space of Schwartz sections of $E$ and by $\mathcal{S}^{*}(X, E)$ its dual space.

See [Aizenbud and Lapid 2012, p. 309] for more details.

Suppose $X$ is a smooth manifold with $G$ acting on $X$. Recall that $X=\bigcup_{i=1}^{l} X_{i}$ is called a $G$-invariant stratification if all sets $X_{i}$ are $G$-invariant and there is some reordering $X_{i_{1}}, X_{i_{2}}, \ldots, X_{i_{l}}$ of $X_{1}, \ldots, X_{l}$ such that all the sets

$$
X_{i_{1}}, X_{i_{1}} \cup X_{i_{2}}, \ldots, X_{i_{1}} \cup X_{i_{2}} \cup \cdots \cup X_{i_{k}}, \ldots, X=X_{i_{1}} \cup \cdots \cup X_{i_{l}}
$$

are open in $X$.

Lemma 2.1. Let a real algebraic Lie group $G$ act on a real algebraic smooth manifold $X$. Let $X=\bigcup_{i=1}^{l} X_{i}$ be a $G$-invariant stratification, and let $\chi$ be a character of $G$. If

$$
\mathcal{S}^{*}(X)^{G, \chi} \neq 0,
$$

then there exist an $1 \leq i \leq l$ and $k \geq 0$ such that

$$
\mathcal{S}^{*}\left(X_{i}, \operatorname{Sym}^{k}\left(C N_{X_{i}}^{X}\right)\right)^{G, \chi} \neq 0 .
$$

This lemma is a special case of [Aizenbud and Lapid 2012, Proposition B.3].

Theorem 2.2 [Aizenbud and Lapid 2012, Theorem B.6]. Let $G$ be a real algebraic group acting transitively on a real algebraic smooth manifold $Z$ and let $\varphi: X \rightarrow Z$ be a G-equivariant smooth map. Fix $z \in Z$ and let $X_{z}$ be the fiber of $z$. Let $\chi$ be a tempered character of $G$ [Aizenbud et al. 2008, Definition 5.1.1], and let $\delta_{G}$ and $\delta_{G_{z}}$ be the modulus characters of the groups $G$ and $G_{z}$ respectively. Then $S^{*}(X)^{G, \chi}$ is canonically isomorphic to $S^{*}\left(X_{z}\right)^{G_{z}, \chi \delta_{G_{z}}^{-1} \delta_{G}}$.

Moreover, for any $G$-equivariant bundle $E$ on $X$, the space $S^{*}(X, E)^{G, \chi}$ is canonically isomorphic to $S^{*}\left(X_{z}, E \mid X_{z}\right)^{G_{z}, \chi \delta_{G_{z}}^{-1} \delta_{G}}$.

\section{Some matrix spaces decompositions}

In this section we obtain some matrix space decompositions that will be used in this work. In the following lemma we analyze the structure of orbits of the action of the Borel subgroup $B_{n}$ on $X_{n}$. Let $W_{n}=S_{n}$ be the Weyl group of $G_{n}(\mathbb{C})$.

Lemma 3.1. There is a bijection between $B_{n} \backslash X_{n}=B_{n} \backslash G_{n}(\mathbb{C}) / G_{n}(\mathbb{R})$ and the space of involutions $W_{n, 2}=\left\{w \in W_{n}: w^{2}=1\right\}$.

Proof. Recall that $X_{n}=\left\{x \in G_{n}(\mathbb{C}): x \cdot \bar{x}=I\right\}$ and let $x \in X_{n}$. Let

$$
T=\left\{\operatorname{diag}\left(d_{1}, \ldots, d_{n}\right): d_{i} \in \mathbb{C}^{*} \text { for all } i\right\}
$$


be a maximal torus in $G_{n}(\mathbb{C})$. From [Lapid and Rogawski 2003, Lemma 4.1.1] (see also [Springer 1984]), the $B_{n}$-orbit of $x$ intersects the normalizer

$$
N(T):=\left\{g \in G_{n}(\mathbb{C}): g T g^{-1}=T\right\} .
$$

It is a well-known fact that $N(T)=\left\{d \cdot w: d \in T, w \in W_{n}\right\}$. Thus, we may assume $x=d w$, where $w \in W_{n}$ and $d=\operatorname{diag}\left(d_{1}, d_{2}, \ldots, d_{n}\right)$. Note that $w$ is uniquely determined by $x$. Since $x \cdot \bar{x}=I$, we have $d w=w^{-1} \bar{d}^{-1}$. We obtain $w=w^{-1}$ and therefore $w^{2}=1$, i.e., $w \in W_{n, 2}$.

Therefore, we can assume that $w \in W_{n, 2}$ in the decomposition $x=d w$. On the other hand, it is clear that different involutions $w, w^{\prime} \in W_{n, 2}$ belong to disjoint orbits of $B_{n}$. Indeed, $l(b) w:=b w \bar{b}^{-1} \neq w^{\prime}$ for all $b \in B_{n}$.

It remains to show that the $B_{n}$-orbit of $x=d w$ contains the point $w$, i.e., there is some $b \in B_{n}$ such that $l(b) x=w$. Since $w$ is an involution it is enough to check the claim for $1 \times 1$ and $2 \times 2$ matrices. For the $1 \times 1$ matrix $x=(b)_{1 \times 1}$, the assumption $x \bar{x}=I$ gives $b \bar{b}=1$, and we want to prove that $b=\mu \bar{\mu}^{-1}$. Clearly, there is such a $\mu$.

For a $2 \times 2$ matrix of the form $b=\left(\begin{array}{cc}d_{1} & 0 \\ 0 & d_{2}\end{array}\right)$, the assumption

$$
x=b\left(\begin{array}{ll}
0 & 1 \\
1 & 0
\end{array}\right)=\left(\begin{array}{cc}
0 & d_{1} \\
d_{2} & 0
\end{array}\right) \in X
$$

gives the condition $d_{1} \bar{d}_{2}=1$ on the entries $d_{1}, d_{2}$. We seek an invertible matrix $\left(\begin{array}{cc}\mu_{1} & 0 \\ 0 & \mu_{2}\end{array}\right)$ such that

$$
\left(\begin{array}{cc}
\mu_{1} & 0 \\
0 & \mu_{2}
\end{array}\right)\left(\begin{array}{cc}
0 & d_{1} \\
d_{2} & 0
\end{array}\right)\left(\begin{array}{cc}
\bar{\mu}_{1}^{-1} & 0 \\
0 & {\overline{\mu_{2}}}^{-1}
\end{array}\right)=\left(\begin{array}{ll}
0 & 1 \\
1 & 0
\end{array}\right)
$$

Matrix multiplication gives the condition $d_{1} \mu_{1}{\overline{\mu_{2}}}^{-1}=1$, and clearly there are such a $\mu_{1}$ and $\mu_{2}$.

In the next paragraph let us fix $n$ and define $G=G_{n}(\mathbb{C}), H=G_{n}(\mathbb{R})$. Our goal is to obtain a generalized Cartan decomposition $G=K A H$, where $K$ is a maximal compact subgroup of $G$ consisting of all unitary matrices in $G$ and $A$ is a torus which we will now describe. Let $m=[n / 2]$. Note that $H=G^{\sigma}$ and $K=G^{\tau}$, where $\sigma(g)=\bar{g}$ and $\tau(g)=g^{*}={ }^{t} \bar{g}$. Let $\mathfrak{g}$ be the Lie algebra of $G$ over the field $\mathbb{C}$. Following [Kobayashi 2007, Fact 2.1, p. 7], we take a to be a maximal abelian subspace in

$$
\mathfrak{g}^{-\sigma,-\tau}=\{X \in \mathfrak{g}: \tau X=\sigma X=-X\} .
$$

Following this recipe, let us define

$$
\mathfrak{a}=\sum_{j=1}^{m} i \mathbb{R}\left(E_{2 j+1,2 j}-E_{2 j, 2 j+1}\right) .
$$


Recall that

$$
\exp \left(\begin{array}{cc}
0 & i t \\
-i t & 0
\end{array}\right)=\left(\begin{array}{cc}
\operatorname{ch}(t) & i \operatorname{sh}(t) \\
-i \operatorname{sh}(t) & \operatorname{ch}(t)
\end{array}\right)
$$

and let $A$ be the Lie group corresponding to $\mathfrak{a}$. Denote by $a\left(t_{1}, t_{2}, \ldots, t_{m}\right)$ the $n \times n$

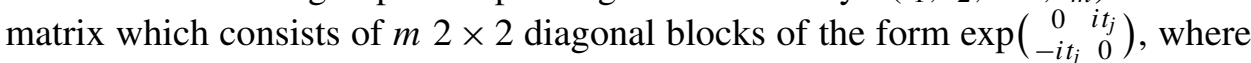
$j=1,2, \ldots, m$ if $n=2 m$ is even, and which consists of these blocks and $a_{n n}=1$ in the last diagonal place if $n=2 m+1$ is odd. For example, if $n=4$ then

$$
a\left(t_{1}, t_{2}\right)=\left(\begin{array}{cccc}
\cosh \left(t_{1}\right) & i \sinh \left(t_{1}\right) & 0 & 0 \\
-i \sinh \left(t_{1}\right) & \cosh \left(t_{1}\right) & 0 & 0 \\
0 & 0 & \cosh \left(t_{2}\right) & i \sinh \left(t_{2}\right) \\
0 & 0 & -i \sinh \left(t_{2}\right) & \cosh \left(t_{2}\right)
\end{array}\right) .
$$

We have

$$
A=\left\{a\left(t_{1}, t_{2}, \ldots, t_{m}\right): t_{1}, t_{2}, \ldots, t_{m} \in \mathbb{R}\right\}
$$

Define

$$
A^{+}=\left\{a\left(t_{1}, t_{2}, \ldots, t_{m}\right): t_{1} \geq t_{2} \geq \cdots \geq t_{m} \geq 0\right\} .
$$

Theorem 3.2. There is a decomposition $G=K A^{+} H$. That is, every element $g \in G$ can be written as

$$
g=k a h, \quad \text { where } k \in K, a \in A^{+}, h \in H .
$$

Moreover, the $a \in A^{+}$in decomposition (3-2) is uniquely determined by $g$.

Remark 3.3. By taking the transpose of (3-2) we obtain a similar decomposition $G=H A^{+} K$. That is, every $g \in G$ can be written as

$$
g=h a k, \quad \text { where } h \in H, a \in A^{+}, k \in K,
$$

and $a \in A^{+}$in this decomposition is uniquely determined by $g$. Actually, after taking the transpose of (3-2), we obtain that $a^{t} \in A$ and in general $a^{t} \notin A^{+}$. But the permutation group $S_{n}$ is naturally contained in both $K$ and $H$ and we can replace $a \in A$ with $a^{\prime}=w_{1} a w_{2}$ such that $a^{\prime} \in A^{+}$.

Proof of Theorem 3.2. To prove the existence part we will show that $G=K A H$. Since permutation matrices are clearly in $H \cap K$, the equality $G=K A^{+} H$ will easily follow from the equality $G=K A H$. Let $g \in G$. Our goal is to achieve a decomposition $g=k a h$ with $h \in H, a \in A$, and $k \in K$. Suppose that $g$ is of such a form. Then, since $h^{*}={ }^{t} h, a^{*}=a$, and $k^{*}=k^{-1}$ we get

$$
g^{*} g={ }^{t} h a^{2} h, \quad h \in H, a \in A .
$$

On the other hand, suppose that every matrix of the form $g^{*} g$ can be written as (3-4). Then write $g=\left(\left(g^{*}\right)^{-1 t} h a\right) a h$, and let us show that $k=\left(g^{*}\right)^{-1 t} h a$ is a unitary 
matrix. Indeed,

$$
k^{*} k=a^{*}\left({ }^{t} h\right)^{*}\left(\left(g^{*}\right)^{-1}\right)^{*}\left(g^{*}\right)^{-1 t} h a=a h\left(g^{*} g\right)^{-1 t} h a=a h\left({ }^{t} h a^{2} h\right)^{-1 t} h a=I .
$$

Therefore, to prove the existence part of the theorem, it is enough to prove that every matrix of the form $g^{*} g$ can be written in the form (3-4). For this purpose write $g^{*} g=x+i y, x, y \in H$. Then $x={ }^{t} x$ is symmetric, and $y=-{ }^{t} y$ is antisymmetric. Also, ${ }^{t} v g^{*} g v>0$ for every $0 \neq v \in \mathbb{R}^{n}$. Hence, $x$ is a positive definite matrix; that is, ${ }^{t} v x\left({ }^{t} x\right) v={ }^{t} v g^{*} g v>0$ for every $0 \neq v \in \mathbb{R}^{n}$. Thus, there is a matrix $h \in H$ such that ${ }^{t} h x h=I$. Then ${ }^{t} h g^{*} g h=I+i\left({ }^{t} h y h\right)$. The matrix $z:={ }^{t} h y h$ is antisymmetric and it is a standard fact in linear algebra that it is diagonalizable by a real orthogonal matrix. Consequently, $h^{\prime} z h^{-1}=d$, with $d$ consisting of $m=\lfloor n / 2\rfloor 2 \times 2$ blocks of the form

$$
\left(\begin{array}{cc}
d_{2 i-1,2 i-1} & d_{2 i-1,2 i} \\
d_{2 i, 2 i-1} & d_{2 i, 2 i}
\end{array}\right)=\left(\begin{array}{cc}
0 & \lambda_{i} \\
-\lambda_{i} & 0
\end{array}\right)
$$

in the case when $n$ is even, and $m$ such blocks and the last row zero in the case when $n$ is odd. Note also that the numbers $\lambda_{i}$ are uniquely determined up to a permutation by the matrix $g g^{*}$ since they are eigenvalues of $h y^{t} h$. Clearly, every $2 \times 2$ block of the form $\left(\begin{array}{cc}1 & i \lambda \\ -i \lambda & 1\end{array}\right)$ can be transformed by a diagonal matrix $\left(\begin{array}{cc}d_{1} & 0 \\ 0 & d_{2}\end{array}\right)$ to the form

$$
\left(\begin{array}{cc}
\operatorname{ch}(\mu) & i \operatorname{sh}(\mu) \\
-i \operatorname{sh}(\mu) & \operatorname{ch}(\mu)
\end{array}\right)=\exp \left(\mu\left(\begin{array}{cc}
0 & i \\
-i & 0
\end{array}\right)\right)
$$

Taking in every block $a$ of the form $\exp \left(\frac{\mu}{2}\left(\begin{array}{cc}0 & i \\ -i & 0\end{array}\right)\right)$ proves the existence of the decomposition $g^{*} g={ }^{t} h a^{2} h$ and thus establishes the existence of the decomposition $g=k a h$.

We now prove the uniqueness part of the theorem. Note that $H$ acts on the space of positive definite matrices of the form $g^{*} g$ by $h \cdot x:=h^{t} x h$. Let us take $h, b, c \in H$ and suppose $h \cdot(I+i b)=I+i c$. Then $h$ is an orthogonal matrix, ${ }^{t} h h=I$, and thus $c=h^{-1} b h$. In particular, the eigenvalues of $b$ and $c$ are equal. Now, to prove the uniqueness of $a \in A^{+}$in the decomposition (3-2) let us write $a=\operatorname{Re}(a)+i \operatorname{Im}(a)$ and note that $H \cdot a=H \cdot(I+i \operatorname{Im}(a))$. Since the eigenvalues of $i \operatorname{Im}(a)$ are $\pm \sinh \left(\lambda_{1}\right), \ldots, \pm \sinh \left(\lambda_{n}\right)$ we see that if $a_{1}, a_{2} \in A^{+}$and $a_{1} \neq a_{2}$, then $H \cdot\left(I+i \operatorname{Im}\left(a_{1}\right)\right) \neq H \cdot\left(I+i \operatorname{Im}\left(a_{2}\right)\right)$, and therefore $H \cdot a_{1} \neq H \cdot a_{2}$. It follows that the $a^{2} \in A^{+}$part in $g^{*} g={ }^{t} h a^{2} h$ is uniquely determined by $g$. As a result, $a \in A^{+}$is uniquely determined by $g$.

\section{Proof of Theorem 1.2}

In this paragraph $n$ is fixed and $G=G_{n}(\mathbb{C}), H=G_{n}(\mathbb{R})$, and $B=B_{n}(\mathbb{C})$. We denote by $M$ the standard maximal torus in $G$ and by $W_{2}=W_{n, 2}$ the set of involutions 
in $S_{n}$. As a starting point of the proof, observe that

$$
I(\chi)^{*}=\mathcal{S}^{*}(G)^{B, \chi \delta_{0}^{-\frac{1}{2}}},
$$

where $B$ acts on the space of tempered distributions $\mathcal{S}^{*}(G)$ from the left. We have

$$
\operatorname{Hom}_{H}(I(\chi), \mathbb{C})=\mathcal{S}^{*}(G / H)^{B, \chi \delta_{0}^{-\frac{1}{2}}} .
$$

We will stratify $X:=G / H$ by $B$-orbits. By Lemma 3.1, we have $B \backslash X=W_{2}$. Suppose $\operatorname{Hom}_{H}(I(\chi), \mathbb{C}) \neq 0$. By Lemma 2.1 there exists an involution $w \in W_{2}$ and a $k \geq 0$ such that

$$
\mathcal{S}^{*}\left(B(w), \operatorname{Sym}^{k}\left(C N_{B(w)}^{X}\right)\right)^{B, \chi \delta_{0}^{-\frac{1}{2}}} \neq 0 .
$$

Note that $B$ acts on $B(w)$ transitively, the stabilizer of $w$ under the action of $B$ is $B_{w}$, and $\left.\delta_{0}^{1 / 2}\right|_{B^{w}}=\delta_{B^{w}}$. Therefore, by Frobenius reciprocity (Theorem 2.2),

$$
\begin{aligned}
\mathcal{S}^{*}\left(B(w), \operatorname{Sym}^{k}\left(C N_{B(w)}^{X}\right)\right)^{B, \chi \delta_{0}^{-\frac{1}{2}}} & =\mathcal{S}^{*}\left(\{w\}, \operatorname{Sym}^{k}\left(C N_{B(w), w}^{X}\right)\right)^{B^{w}, \chi \delta_{0}^{-\frac{1}{2}} \delta_{B^{w}}^{-1} \delta_{0}} \\
& =\mathcal{S}^{*}\left(\{w\}, \operatorname{Sym}^{k}\left(C N_{B(w), w}^{X}\right)\right)^{B^{w}, \chi} \\
& =\left(\operatorname{Sym}^{k}\left(N_{B(w), w}^{X}\right) \otimes_{\mathbb{R}} \mathbb{C}\right)^{B^{w}, \chi} .
\end{aligned}
$$

Observe that $M^{w} \subset B^{w}$. Hence $\left(\operatorname{Sym}^{k}\left(N_{B(w), w}^{X}\right) \otimes_{\mathbb{R}} \mathbb{C}\right)^{B^{w}, \chi} \neq 0$ implies

$$
\left(\operatorname{Sym}^{k}\left(N_{B(w), w}^{X}\right) \otimes_{\mathbb{R}} \mathbb{C}\right)^{M^{w}, \chi} \neq 0 .
$$

Note that

$$
\begin{aligned}
M^{w} & =\left\{t \in M: t^{-1} w \bar{t}=w\right\}=\{t \in M: t=w \bar{t} w\} \\
& =\left\{t=\operatorname{diag}\left(t_{1}, t_{2}, \ldots, t_{n}\right) \in M: t_{i}=\overline{t_{w(i)}} \text { for } 1 \leq i \leq n\right\} .
\end{aligned}
$$

It will be useful to obtain one more formula for $M^{w}$. It is easy to see, by examining the case of $1 \times 1$ and $2 \times 2$ matrices, that

$$
\begin{aligned}
& M^{w}=\left\{t(w \bar{t} w) a: t \in M, a=\operatorname{diag}\left(a_{1}, a_{2}, \ldots, a_{n}\right),\right. \\
& \left.\quad a_{i}=1 \text { if } w(i) \neq i, a_{i}= \pm 1 \text { if } w(i)=i\right\} .
\end{aligned}
$$

In the next lemma we perform a calculation of the normal space $N_{B(w), w}^{X}$. Note that this is a finite-dimensional vector space over $\mathbb{R}$. Since the group $M^{w}$ preserves the tangent space $T_{w}^{B(w)}$ and clearly preserves the tangent space $T_{w}^{X}$, there is an action of $M^{w}$ on the normal space $N_{B(w), w}^{X}$. By taking the scalar extension $N_{B(w), w}^{X} \otimes \mathbb{C}$, we get a complex representation of $M^{w}$. Since $M^{w}$ is abelian, this representation decomposes into a direct sum of irreducible, one-dimensional representations. 
Lemma 4.1. We have

$$
N_{B(w), w}^{X} \otimes_{\mathbb{R}} \mathbb{C}=\bigoplus_{\left\{(i, j) \in I_{w}\right\}} \alpha_{\delta(i, j)}
$$

as a representation of $M^{w}$.

Before proving this lemma, we give the following corollary.

Corollary 4.2. We have

$$
\operatorname{Sym}\left(N_{B(w), w}^{X} \otimes_{\mathbb{R}} \mathbb{C}\right)=\bigoplus_{\kappa: I_{w} \rightarrow \mathbb{Z}_{\geq 0}} \alpha_{\kappa}
$$

as a representation of $M^{w}$.

Proof of Lemma 4.1. Let us denote by $e_{i, j}$ the elementary matrix with 1 at the $(i, j)$-th entry and zeros in all other entries. The tangent space of $X$ at $w$ is equal to

$$
\begin{aligned}
T_{w}^{X} & =\left\{A \in \operatorname{Mat}_{n}(\mathbb{C}): A w+w \bar{A}=0\right\}=\left\{A \in \operatorname{Mat}_{n}(\mathbb{C}): w A w=-\bar{A}\right\} \\
& =\operatorname{Span}_{\mathbb{B}}\left\{-e_{i, j}+e_{w(i), w(j)}, \sqrt{-1}\left(e_{i, j}+e_{w(i), w(j)}\right): 1 \leq i, j \leq n\right\} .
\end{aligned}
$$

On the other hand,

$$
T_{w}^{B(w)}=\left\{-A w+w \bar{A}: A \in \operatorname{Mat}_{n}(\mathbb{C}), A \text { is upper triangular }\right\} .
$$

Since $e_{i, j} w=e_{i, w(j)}$ and $w e_{i, j}=e_{w(i), j}$, we obtain that

$$
\begin{aligned}
T_{w}^{B(w)} & =\operatorname{Span}_{\mathbb{R}}\left\{-e_{i, w(j)}+e_{w(i), j}, \sqrt{-1}\left(e_{i, w(j)}+e_{w(i), j}\right): 1 \leq i \leq j \leq n\right\} \\
& =\operatorname{Span}_{\mathbb{R}}\left\{-e_{i, j}+e_{w(i), w(j)}, \sqrt{-1}\left(e_{i, j}+e_{w(i), w(j)}\right): i \leq w(j)\right\} \\
& =\operatorname{Span}_{\mathbb{C}}\left\{e_{i, j}, e_{w(i), w(j)}: i \leq w(j)\right\} \cap T_{w}^{X} \\
& =\operatorname{Span}_{\mathbb{C}}\left\{e_{i, j}: i \leq w(j) \text { or } j \geq w(i)\right\} \cap T_{w}^{X} .
\end{aligned}
$$

Hence

$$
\begin{aligned}
N_{B(w), w}^{X} & \cong \operatorname{Span}_{\mathbb{C}}\left\{e_{i, j}: i>w(j), w(i)>j\right\} \cap T_{w}^{X} \\
& =\operatorname{Span}_{\mathbb{C}}\left\{e_{i, w(j)}: i>j, w(i)>w(j)\right\} \cap T_{w}^{X} \\
& =\operatorname{Span}_{\mathbb{C}}\left\{e_{i, w(j)}:(i, j) \in I_{w}\right\} \cap T_{w}^{X} .
\end{aligned}
$$

Let us denote $V=\operatorname{Span}_{\mathbb{C}}\left\{e_{i, w(j)}:(i, j) \in I_{w}\right\}$. Note that if $e_{i, w(j)} \in V$ then also $e_{w(i), j} \in V$, since $w$ is an involution and for an involution

$$
(i, j) \in I_{w} \Longleftrightarrow(w(i), w(j)) \in I_{w} .
$$


Let us use the lexicographic ordering on pairs $(i, j)$ : write $(i, j)<\left(i^{\prime}, j^{\prime}\right)$ if $i<i^{\prime}$ or if $i=i^{\prime}$ and $j<j^{\prime}$. Then we may rewrite (4-2) as

$$
\begin{array}{r}
N_{B(w), w}^{X} \cong \operatorname{Span}_{\mathbb{R}}\left\{\sqrt{-1} e_{i, w(j)}:(i, j) \in I_{w},(i, j)=(w(i), w(j))\right\} \\
\oplus \operatorname{Span}_{\mathbb{R}}\left\{e_{i, w(j)}-e_{w(i), j}, \sqrt{-1}\left(e_{i, w(j)}+e_{w(i), j}\right):\right. \\
\left.\quad(i, j) \in I_{w},(i, j)<(w(i), w(j))\right\} .
\end{array}
$$

For $t=\operatorname{diag}\left(t_{1}, \ldots, t_{n}\right) \in M$ we have

$$
t e_{i, j} \bar{t}^{-1}=\left(t_{i} / \bar{t}_{j}\right) e_{i, j},
$$

and for $t \in M^{w}$ we also have

$$
t e_{i, w(j)} \bar{t}^{-1}=\left(t_{i} / \overline{t_{w(j)}}\right) e_{i, w(j)}=\left(t_{i} / t_{j}\right) e_{i, w(j)} .
$$

Therefore, the action of $M^{w}$ on $e_{i, w(j)}$ is given by $\alpha_{\delta(i, j)}$. We obtain that, as a representation of $M^{w}$, $N_{B(w), w}^{X} \otimes_{\mathbb{R}} \mathbb{C}$

$$
\begin{aligned}
& \cong \bigoplus_{\left\{(i, j) \in I_{w}:(i, j)=(w(i), w(j))\right\}} \alpha_{\delta(i, j)} \oplus \bigoplus_{\left\{(i, j) \in I_{w}:(i, j)<(w(i), w(j))\right\}}\left(\alpha_{\delta(i, j)} \oplus \alpha_{\delta(w(i), w(j))}\right) \\
& =\bigoplus_{\left\{(i, j) \in I_{w}:(i, j)=(w(i), w(j))\right\}} \alpha_{\delta(i, j)} \oplus \bigoplus_{\left\{(i, j) \in I_{w}:(i, j)<(w(i), w(j))\right\}} \alpha_{\delta(i, j)} \oplus \bigoplus_{\left\{(i, j) \in I_{w}:(i, j)>(w(i), w(j))\right\}} \alpha_{\delta(i, j)} \\
& =\bigoplus_{\left\{(i, j) \in I_{w}\right\}} \alpha_{\delta(i, j)} .
\end{aligned}
$$

Lemma 4.3. If $\left(\operatorname{Sym}^{k}\left(N_{B(w), w}^{X}\right) \otimes_{\mathbb{R}} \mathbb{C}\right)^{M^{w}, \chi} \neq 0$ then $k=0, w(\chi)=\bar{\chi}^{-1}$, and $\chi_{i}(-1)=1$ for all $1 \leq i \leq n$ such that $w(i)=i$.

Proof. Note that, for $t \in M$ and $w \in W_{2}$, the element $w t w$ is also diagonal and its diagonal entries are the permutation of diagonal entries of $t$ by $w$, i.e.,

$$
(w t w)_{i i}=t_{w(i), w(i)} .
$$

By (4-1), if $\left.\alpha_{\kappa}\right|_{M^{w}}=\left.\chi\right|_{M^{w}}$, then for every $t \in M$ we have

$$
\left.\alpha_{\kappa}(t(w \bar{t} w))=\alpha_{\kappa}(t) \overline{w\left(\alpha_{\kappa}\right)(t)}=\chi(t) \overline{\chi(w(t)}\right)=\chi(t(w \bar{t} w)) .
$$

That is,

$$
\left(\left.\alpha_{\kappa}\right|_{M^{w}}=\left.\chi\right|_{M^{w}}\right) \Rightarrow\left(\overline{\alpha_{\kappa}} w\left(\alpha_{\kappa}\right)=\bar{\chi} w(\chi)\right) .
$$

To obtain (4-4) just put $a=1$ in (4-1). The set of $\kappa$ s that satisfy

$$
\overline{\alpha_{\kappa}} w\left(\alpha_{\kappa}\right)=\bar{\chi} w(\chi)
$$


is $\{\kappa \equiv 0\}$ if $w(\chi)=\overline{\chi^{-1}}$ and is empty otherwise. Indeed, we take the absolute value on both sides of (4-5) to obtain

$$
\prod_{(i, j) \in I_{w}}\left|\frac{t_{i}}{t_{j}}\right|^{\kappa(i, j)+\kappa(w(i), w(j))}=\prod_{i=1}^{n}\left|t_{i}\right|^{\lambda_{i}+\lambda_{w(i)}} .
$$

First, we will deduce from the last equation that the right-hand side of this equation is 1 . Note that from (4-6) it follows, by substituting $t_{i}=c$ for all $i$ with a generic $c \in \mathbb{C}^{*}$, that

$$
\lambda_{1}+\cdots+\lambda_{n}=0
$$

Since no pair $(1, i)$ belongs to $I_{w}$, it follows that $\lambda_{1}+\lambda_{w(1)} \leq 0$. Let $i$ be the first index such that $\lambda_{i}+\lambda_{w(i)}>0$. Then on the left-hand side of (4-6) the power of $\left|t_{i}\right|$ is positive, thus there is a $j$ such that $(i, j) \in I_{w}$. Hence $i>j, w(i)>w(j)$ and from the assumption $\lambda_{1} \geq \lambda_{2} \geq \cdots \geq \lambda_{n}$ we obtain $\lambda_{i} \leq \lambda_{j}$ and $\lambda_{w(i)} \leq \lambda_{w(j)}$. Thus

$$
0<\lambda_{i}+\lambda_{w(i)} \leq \lambda_{j}+\lambda_{w(j)} \leq 0,
$$

a contradiction! Therefore, for every $i$, there is an inequality $\lambda_{i}+\lambda_{w(i)} \leq 0$. Since the sum of all $\lambda \mathrm{s}$ is equal to 0 , we obtain $\lambda_{i}+\lambda_{w(i)}=0$ for every $i$. Hence, $\lambda_{1} \geq 0$ and $\lambda_{n} \leq 0$.

Now, we can deduce $\kappa \equiv 0$. Let $j$ be the minimal index such that there exists a pair $(i, j) \in I_{w}$ with the property

$$
\kappa(i, j) \neq 0 \quad \text { or } \quad \kappa(w(i), w(j)) \neq 0 .
$$

The power of $\left|t_{j}\right|$ on the right-hand side of (4-5) must equal 0 , thus there is a pair $(j, k) \in I_{w}$ such that $\kappa(j, k) \neq 0$ or $\kappa(w(j), w(k)) \neq 0$. In both cases we obtain a contradiction to the minimality of $j$. As a conclusion, we obtain that (4-5) implies $\kappa \equiv 0$.

Suppose now that $w(\chi)=\overline{\chi^{-1}}$ and thus $\kappa \equiv 0$. Then $\alpha_{\kappa}=1$, the identity character. We want to prove that $\chi_{i}(-1)=1$ for all $i$ such that $w(i)=i$. This follows from $\chi(a)=\alpha_{\kappa}(a)=1$ for $a=\operatorname{diag}\left(a_{1}, \ldots, a_{n}\right)$, where $a_{i}= \pm 1$ whenever $w(i)=i$ and $a_{i}=1$ otherwise.

\section{Calculation of Rankin-Selberg gamma factors}

In this section we recall the notion of Rankin-Selberg integrals and apply the results of previous sections to calculate special values of Rankin-Selberg gamma factors. The exposition and notation follows [Jacquet 2009]. Let $\chi: B_{n} \rightarrow \mathbb{C}^{\times}$be a multiplicative character and let $\lambda: I(\chi) \rightarrow \mathbb{C}$ be a $\psi$-form on $I(\chi)$. Recall that such a $\lambda$ always exists and it is unique up to a scalar multiple. If $f \in V, g \in G_{n}$, we set

$$
W_{f}(g)=\lambda(R(g) f) .
$$


Let $\mathcal{W}(I(\chi), \psi)$ be the space spanned by the functions of the form $W_{f}$.

For every $n$, we denote by $w_{n}$ the $n \times n$ permutation matrix whose antidiagonal entries are 1. If $n>n^{\prime}$, we define

$$
w_{n, n^{\prime}}=\left(\begin{array}{cc}
1_{n^{\prime}} & 0 \\
0 & w_{n-n^{\prime}}
\end{array}\right) .
$$

If $f \in I(\chi)$, then the function $\tilde{f}$ is defined by

$$
\tilde{f}(g):=f\left(w_{n}{ }^{t}{ }^{-1}\right) .
$$

Let $\pi$ be an irreducible representation of $G_{n}(\mathbb{C})$ and let $\pi^{\prime}$ be an irreducible representation of $G_{m}(\mathbb{C})$. Suppose $\pi$ is the Langlands quotient of $I(\chi)$ and $\pi^{\prime}$ is the Langlands quotient of $I\left(\chi^{\prime}\right)$. We choose a $\psi$-form $\lambda$ on $I(\chi)$ and a $\bar{\psi}$-form $\lambda^{\prime}$ on $I\left(\chi^{\prime}\right)$. Rankin-Selberg integrals are defined as follows. For $f \in I(\chi), f^{\prime} \in I\left(\chi^{\prime}\right)$, set

$$
W=W_{f}, \quad W^{\prime}=W_{f^{\prime}} .
$$

For $W=W_{f}$, set

$$
\widetilde{W}_{f}:=W_{f}\left(w_{n}{ }^{t} g^{-1}\right) .
$$

Note that $\widetilde{W}_{f}(g)=W_{\tilde{f}}(g)$ and $W_{\tilde{f}}(g) \in \mathcal{W}\left(I\left(\chi^{-1}\right), \bar{\psi}\right)$.

If $n>n^{\prime}$, we set

$$
\Psi\left(s, W, W^{\prime}\right)=\int W\left(\begin{array}{cc}
g & 0 \\
0 & 1_{n-n^{\prime}}
\end{array}\right) W^{\prime}(g)|\operatorname{det} g|_{\mathbb{C}}^{s-\frac{n-n^{\prime}}{2}} d g .
$$

In addition, for $0 \leq j \leq n-n^{\prime}-1$, we set

$$
\Psi_{j}\left(s, W, W^{\prime}\right)=\int W\left(\begin{array}{ccc}
g & 0 & 0 \\
X & 1_{j} & 0 \\
0 & 0 & 1_{n-n^{\prime}-j}
\end{array}\right) W^{\prime}(g)|\operatorname{det} g|_{\mathbb{C}}^{s-\frac{n-n^{\prime}}{2}} d X d g .
$$

Here $X$ is integrated over the space $M(m \times j, \mathbb{C})$ of matrices with $m$ rows and $j$ columns. In each integral, $g$ is integrated over the quotient $U_{n}(\mathbb{C}) \backslash G_{n}(\mathbb{C})$.

If $n=n^{\prime}$, we let $\Phi$ be a Schwartz function on $\mathbb{C}^{n}$ and we set

$$
\Psi\left(s, W, W^{\prime}, \Phi\right)=\int W(g) W^{\prime}(g) \Phi((0,0, \ldots, 0,1) g)|\operatorname{det} g|_{\mathbb{C}}^{s} d g .
$$

The Rankin-Selberg gamma factor $\gamma\left(s, \pi \times \pi^{\prime}, \psi\right)$ is a proportionality factor appearing in functional equations on certain Rankin-Selberg integrals. We quote here [Jacquet 2009, Theorem 2.1]. 
Theorem 5.1. (1) The integrals (5-1), (5-2), and (5-3) converge for $\operatorname{Re}(s) \gg 0$.

(2) Each integral extends to a meromorphic function of s which is a holomorphic multiple of $L\left(s, \pi \times \pi^{\prime}\right)$ bounded at infinity in vertical strips. See [Jacquet 2009] for the definition of $L\left(s, \pi \times \pi^{\prime}\right)$.

(3) The following functional equations are satisfied. If $n>n^{\prime}$,

$$
\begin{aligned}
& \Psi\left(1-s, \widetilde{W}, \widetilde{W^{\prime}}\right)=\omega_{I(\chi)}(-1)^{n-1} \omega_{I\left(\chi^{\prime}\right)}(-1) \gamma\left(s, I(\chi) \times I\left(\chi^{\prime}\right), \psi\right) \Psi\left(s, W, W^{\prime}\right) . \\
& \text { If } n>n^{\prime}+1 \text { and } \beta=n-n^{\prime}-1-j,
\end{aligned}
$$$$
\Psi_{j}\left(1-s, \pi\left(w_{n, n^{\prime}}\right) \widetilde{W}, \widetilde{W^{\prime}}\right)=\omega_{I(\chi)}(-1)^{n^{\prime}} \omega_{I\left(\chi^{\prime}\right)}(-1) \gamma\left(s, \pi \times \pi^{\prime}, \psi\right) \Psi_{\beta}\left(s, W, W^{\prime}\right) .
$$$$
\text { If } n=n^{\prime} \text {, }
$$$$
\Psi\left(1-s, \widetilde{W}, \widetilde{W}^{\prime}, \hat{\Phi}\right)=\omega_{I(\chi)}(-1)^{n-1} \gamma\left(s, \pi \times \pi^{\prime}, \psi\right) \Psi\left(s, W, W^{\prime}, \Phi\right) .
$$

We will calculate the special values of the Rankin-Selberg gamma factor of $G_{n}(\mathbb{R})$-distinguished representations. The main tool will be the classification of such representations obtained in Theorem 1.2 and basic properties of Rankin-Selberg gamma factors from [Jacquet 2009, Lemma 16.3].

Let us recall some facts about one-dimensional Tate gamma factors. Let $\chi$ be a one-dimensional character $\chi: \mathbb{C}^{*} \rightarrow \mathbb{C}^{*}$. We have the following functional equation for Tate gamma factors:

$$
\gamma(s, \chi, \psi) \gamma\left(1-s, \overline{\chi^{-1}}, \overline{\psi^{-1}}\right)=1 .
$$

Since we assume $\psi$ is trivial on $\mathbb{R}$, we obtain $\overline{\psi^{-1}}=\psi$, and for $s=1 / 2$ we get

$$
\gamma\left(\frac{1}{2}, \chi, \psi\right) \gamma\left(\frac{1}{2}, \overline{\chi^{-1}}, \psi\right)=1
$$

For a real character $\chi$, that is, for $\chi$ satisfying $\chi^{2}=1$, we obtain $\gamma(1 / 2, \chi, \psi)^{2}=1$, and thus $\gamma(1 / 2, \chi, \psi) \in\{1,-1\}$. The value of $\gamma(1 / 2, \chi, \psi)$ depends on $\chi(-1)$. Whenever $\chi(-1)=1$ we obtain

$$
\gamma\left(\frac{1}{2}, \chi, \psi\right)=1
$$

Proof of Theorem 1.1. Recall that if $\pi$ is the Langlands quotient of $I(\chi)$ and $\pi^{\prime}$ is the Langlands quotient of $I\left(\chi^{\prime}\right)$, then

$$
\gamma\left(s, \pi \times \pi^{\prime}, \psi\right)=\gamma\left(s, I(\chi) \times I\left(\chi^{\prime}\right), \psi\right) .
$$

It is well-known that $\chi=\left(\chi_{1}, \ldots, \chi_{t}\right)$, where the $\chi_{i}$ s are one-dimensional characters of $\mathbb{C}$. Similarly, $\chi^{\prime}=\left(\chi_{1}^{\prime}, \ldots, \chi_{r}^{\prime}\right)$, where the $\chi_{i}^{\prime}$ s are one-dimensional 
characters of $\mathbb{C}$. Thus,

(5-7) $\gamma\left(s, I(\chi) \times I\left(\chi^{\prime}\right), \psi\right)=\prod_{i=1}^{t} \gamma\left(s, \chi_{i} \times I\left(\chi^{\prime}\right), \psi\right)=\prod_{i=1}^{t} \prod_{j=1}^{r} \gamma\left(s, \chi_{i} \chi_{j}^{\prime}, \psi\right)$.

Using Theorem 1.2, there exist involutions $w \in S_{t}$ and $w^{\prime} \in S_{r}$ such that $w(\chi)=\bar{\chi}^{-1}$ and $w^{\prime}\left(\chi^{\prime}\right)=\bar{\chi}^{\prime}-1$ and for every fixed point $i$ of $w$, and $j$ of $w^{\prime}$, we have $\chi_{i}(-1)=1$ and $\chi_{j}^{\prime}(-1)=1$. The formula in (5-7) may be rewritten as

$$
\gamma\left(s, I(\chi) \times I\left(\chi^{\prime}\right), \psi\right)=I_{1} I_{2},
$$

where

$$
\begin{aligned}
& I_{1}=\prod_{\left\{(i, j):\left(w(i), w^{\prime}(j)\right)=(i, j)\right\}} \gamma\left(\frac{1}{2}, \chi_{i} \chi_{j}^{\prime}, \psi\right), \\
& I_{2}=\prod_{\left\{(i, j): i<w(i) \text { or }\left(i=w(i) \text { and } w^{\prime}(j)<j\right)\right\}} \gamma\left(\frac{1}{2}, \chi_{i} \chi_{j}^{\prime}, \psi\right) \gamma\left(\frac{1}{2}, \chi_{w(i)} \chi_{w^{\prime}(j)}^{\prime}, \psi\right) .
\end{aligned}
$$

Let us prove that every term appearing in the product $I_{1}$ is 1 . Indeed, by Theorem 1.2 the character $\chi_{i} \chi_{j}^{\prime}$ appearing as the argument in the gamma factor in $I_{1}$ is a real character satisfying $\chi \chi_{j}^{\prime}(-1)=1$, and therefore, by (5-6), we get

$$
\gamma\left(\frac{1}{2}, \chi_{i} \chi_{j}^{\prime}, \psi\right)=1
$$

Each term in the product $I_{2}$ also equals 1 , since $\chi_{w(i)} \chi_{w^{\prime}(j)}^{\prime}=\left(\overline{\chi_{i} \chi_{j}^{\prime}}\right)^{-1}$ and by applying (5-5). Finally, $I_{1}=I_{2}=1$ and we obtain

$$
\gamma\left(\frac{1}{2}, \pi \times \pi^{\prime}, \psi\right)=1
$$

We will need the following technical result about Rankin-Selberg integrals in Section 8 .

Lemma 5.2. Let $(\pi, V),\left(\pi^{\prime}, V^{\prime}\right)$ be generic representations of $G_{n}(\mathbb{C})$ and let

$$
\mathcal{W}(\pi, \psi), \quad \mathcal{W}\left(\pi^{\prime}, \psi^{-1}\right)
$$

be their Whittaker models. Suppose $(\pi, V)$ is unitarizable and $\left(\pi^{\prime}, V^{\prime}\right)$ is tempered. Let $W \in \mathcal{W}(\pi, \psi)), W^{\prime} \in \mathcal{W}\left(\pi^{\prime}, \psi^{-1}\right)$, and $\Phi \in \mathcal{S}\left(\mathbb{C}^{n}\right)$. Then the Rankin-Selberg integral

$$
\int_{U_{n}(\mathbb{C}) \backslash G_{n}(\mathbb{C})} W(g) W^{\prime}(g) \Phi((0,0, \ldots, 0,1) g)|\operatorname{det} g|_{\mathbb{C}}^{s} d g
$$

converges absolutely at $s=1 / 2$. 
Proof. Define

$$
T_{n}=\left\{\operatorname{diag}\left(t_{1}, \ldots, t_{n}\right): t_{i} \in \mathbb{R} \text { and } t_{1} \geq t_{2} \geq \cdots \geq t_{n}>0\right\}
$$

and let $K_{n}$ be a maximal compact subgroup of $G_{n}(\mathbb{C})$ consisting of all unitary matrices in $G_{n}(\mathbb{C})$. Let $\delta$ be the modular character of $B_{n}(\mathbb{C})$. By [Lapid and Mao 2014, Lemma 2.1], we know that there exist a $\lambda>-1 / 2$, a $d>0$ and a continuous seminorm $\mu$ on $\mathcal{W}(\pi, \psi)$ such that

$$
|W(t k)| \leq \delta^{\frac{1}{2}}(t)|\operatorname{det} t|_{\mathbb{C}}^{\lambda}\left|t_{n}\right|_{\mathbb{C}}^{-n \lambda}(1+\|\log t\|)^{d} \mu(W)
$$

for all $t \in T_{n}, k \in K_{n}$ and every $W \in \mathcal{W}(\pi, \psi)$. Similarly, there exist an $\epsilon<0$ such that $\lambda+\epsilon>-1 / 2$, a $d^{\prime}>0$ and a continuous seminorm $\mu^{\prime}$ on $\mathcal{W}\left(\pi^{\prime}, \psi^{-1}\right)$ such that

$$
\left|W^{\prime}(t k)\right| \leq \delta^{\frac{1}{2}}(t)|\operatorname{det} t|_{\mathbb{C}}^{\epsilon}\left|t_{n}\right|_{\mathbb{C}}^{-n \epsilon}(1+\|\log t\|)^{d^{\prime}} \mu^{\prime}\left(W^{\prime}\right)
$$

for all $t \in T_{n}, k \in K_{n}$ and every $W^{\prime} \in \mathcal{W}\left(\pi^{\prime}, \psi^{-1}\right)$. Let us define $\lambda:=\lambda+\epsilon$, $d:=d+d^{\prime}$. All that matters for the estimates is that $\lambda>1 / 2$ and $d>0$. There is a $\phi \in \mathcal{S}(\mathbb{R})$ such that

$$
\begin{aligned}
& \left|W(t k) W^{\prime}(t k) \Phi((0,0, \ldots, 0,1) g)\right| \\
& \quad \leq \delta(t)|\operatorname{det} t|_{\mathbb{C}}^{\lambda}\left|t_{n}\right|_{\mathbb{C}}^{-n \lambda}(1+\|\log t\|)^{d} \phi\left(t_{n}\right) \mu(W) \mu^{\prime}\left(W^{\prime}\right)
\end{aligned}
$$

for all $t \in T_{n}, k \in K_{n}$, every $W \in \mathcal{W}(\pi, \psi)$ and every $W^{\prime} \in \mathcal{W}\left(\pi^{\prime}, \psi^{-1}\right)$. For fixed functions $W, W^{\prime}$ the numbers $\mu(W), \mu^{\prime}\left(W^{\prime}\right)$ are constant and we can move them to the function $\phi$. Thus we can rewrite the last estimate as

$$
\left|W(t k) W^{\prime}(t k) \Phi((0,0, \ldots, 0,1) g)\right| \leq \delta(t)|\operatorname{det} t|_{\mathbb{C}}^{\lambda}\left|t_{n}\right|_{\mathbb{C}}^{-n \lambda}(1+\|\log t\|)^{d} \phi\left(t_{n}\right)
$$

for all $t \in T_{n}, k \in K_{n}$. Let us rewrite the expression

$$
\int_{U_{n}(\mathbb{C}) \backslash G_{n}(\mathbb{C})}\left|W(g) W^{\prime}(g) \Phi((0,0, \ldots, 0,1) g)\right||\operatorname{det} g|_{\mathbb{C}}^{\operatorname{Re}(s)} d g
$$

using the Iwasawa decomposition to obtain

$$
\int_{K_{n}} \int_{T_{n}}\left|W(t k) W^{\prime}(t k) \Phi((0,0, \ldots, 0,1) g)\right||\operatorname{det} t|_{\mathbb{C}}^{\operatorname{Re}(s)} \delta^{-1}(t) d t d k .
$$


For $f: G_{n}(\mathbb{C}) \rightarrow \mathbb{C}$ such that the following integrals are absolutely convergent, we have

$$
\begin{aligned}
\int_{G_{n}(\mathbb{C})} f(g) d g & =\int_{U_{n}(\mathbb{C})} \int_{T_{n}} \int_{K_{n}} f(t u k) d u d t d k \\
& =\iint_{U_{n}(\mathbb{C})} \int_{T_{n}} f\left(\left(t u t^{-1}\right) t k\right) d u d t d k \\
& =\iint_{U_{n}(\mathbb{C})} \int_{T_{n}} \int_{K_{n}} f(u t k) \delta^{-1}(t) d u d t d k .
\end{aligned}
$$

Let us define $\alpha_{i}(t)=t_{i} / t_{i+1}$ for $t \in T_{n}$. Note that

$$
\operatorname{det} t=\prod_{i=1}^{n-1} \alpha_{i}(t)^{i} t_{n}^{n}
$$

The integrand in (5-8) is bounded by

$\left|W(t k) W^{\prime}(t k) \Phi\left(\eta_{n} g\right)\right||\operatorname{det} t|_{\mathbb{C}}^{\operatorname{Re}(s)} \delta^{-1}(t)$

$$
\leq(1+\|\log t\|)^{d} \phi\left(t_{n}\right)\left(\prod_{i=1}^{n-1} \alpha_{i}(t)^{2 i(\operatorname{Re}(s)+\lambda)}\right) t_{n}^{2 n \operatorname{Re}(s)} .
$$

There exists an $e>0$ such that

$$
(1+\|\log t\|)^{d} \leq\left(\prod_{j=1}^{n-1}\left(1+\log \alpha_{j}(t)\right)^{e}\right)\left(1+\log \left|t_{n}\right|\right)^{e}
$$

for all $t \in T_{n}$. We have the estimate

$$
\begin{aligned}
\int_{T_{n}}(1 & +\|\log t\|)^{e} \phi\left(t_{n}\right)|\operatorname{det} t|_{\mathbb{C}(s)+\lambda}^{\operatorname{Re}}\left|t_{n}\right|_{\mathbb{C}}^{-n \lambda} d t \\
& \leq \prod_{j=1}^{n-1} \int_{1}^{\infty}\left(1+\log t_{j}\right)^{e} t_{j}^{2 j(\operatorname{Re}(s)+\lambda)} d^{\times} t_{j} \times \int_{0}^{\infty}\left(1+\left|\log t_{n}\right|\right)^{e} \phi\left(t_{n}\right) t_{n}^{2 n \operatorname{Re}(s)} d^{\times} t_{n} .
\end{aligned}
$$

It follows that the integral absolutely converges for $s$ satisfying $\operatorname{Re}(s)>-\lambda$ and $\operatorname{Re}(s)>0$. As $\lambda>-1 / 2$, we obtain the absolute convergence of the Rankin-Selberg integral at $s=1 / 2$.

\section{Integral representation of Whittaker functions}

Let $n \geq 2$ be fixed and let $K=U_{n}(\mathbb{C})$ be a maximal compact subgroup of $G_{n}(\mathbb{C})$. The next lemma gives a convenient formula for the $G_{n}(\mathbb{R})$-period of a unitary and generic representation $(\pi, V) \in \operatorname{Irr}_{G_{n}(\mathbb{R})}\left(G_{n}(\mathbb{C})\right)$. It is proved in [Lapid and Mao 
2014, Lemma 1.2]. We state it and, for the convenience of the reader, provide a full proof here.

Lemma 6.1 [Lapid and Mao 2014, Lemma 1.2]. Let $(\pi, V) \in \operatorname{Irr}_{G_{n}(\mathbb{R})}\left(G_{n}(\mathbb{C})\right)$ be unitarizable and generic and let $\mathcal{W}(\pi, \psi)$ be its Whittaker model. The functional

$$
\mu: W \mapsto \int_{U_{n-1}(\mathbb{R}) \backslash G_{n-1}(\mathbb{R})} W(h) d h
$$

defines a $P_{n}(\mathbb{R})$-invariant functional on $\mathcal{W}(\pi, \psi)$. Moreover, there exists an $N>0$ and a seminorm $v$ on $\mathcal{W}(\pi, \psi)$ such that for all $g \in G_{n}(\mathbb{C})$ and $W \in \mathcal{W}(\pi, \psi)$ we have the inequality

$$
|\mu(\pi(g) W)| \leq\|g\|_{H}^{N} v(W) .
$$

Proof. By [Wallach 1992, Theorem 15.2.5], there exist a continuous seminorm $v^{\prime}$ on $\mathcal{W}(\pi, \psi)$, a $\lambda>-1 / 2$ and a $d>0$ such that

$$
|W(t k)| \leq \delta^{\frac{1}{2}}(t)|\operatorname{det} t|_{\mathbb{C}}^{\lambda}\left|t_{n}\right|_{\mathbb{C}}^{-n \lambda}(1+\|\log t\|)^{d} v^{\prime}(W)
$$

for every $W \in \mathcal{W}(\pi, \psi), t \in T_{n}$, and $k \in K_{n}$. For $g \in G_{n-1}(\mathbb{R})$ we have $t_{n}=1$. Let us denote

$$
T_{n-1}=\left\{t \in T_{n}: t_{n}=1\right\} .
$$

Let $\delta_{0}(t)$ be the modular character of $B_{n}(\mathbb{R})$. For $t \in B_{n}(\mathbb{R})$ we have that $\delta^{1 / 2}(t)=\delta_{0}(t)$. Multiplying $v^{\prime}$ by a scalar we have the estimate

$$
\begin{aligned}
\int_{U_{n-1}(\mathbb{R}) \backslash G_{n-1}(\mathbb{R})}|W(h)| d h & \leq \int_{K_{n-1}(\mathbb{R})} \int_{T_{n-1}}|W(t k)| \delta_{0}^{-1}(t) d t d k \\
& \leq v^{\prime}(W) \int_{T_{n-1}}|\operatorname{det} t|_{\mathbb{C}}^{\lambda}(1+\|\log t\|)^{d} d t
\end{aligned}
$$

for every $W \in \mathcal{W}(\pi, \psi)$. By the estimates of the previous lemma we obtain that the last integral converges absolutely for $\lambda>-1 / 2$. It follows that $|\mu(W)| \leq \nu^{\prime \prime}(W)$ for a continuous seminorm $v^{\prime \prime}$ on $\mathcal{W}(\pi, \psi)$. Since $\pi$ is of moderate growth, there exist another continuous seminorm $v$ on $\mathcal{W}(\pi, \psi)$ and an $N>0$ such that

$$
|\mu(\pi(g) W)| \leq \nu^{\prime \prime}(\pi(g) W) \leq\|g\|_{H}^{N} \nu(W)
$$

for every $W \in \mathcal{W}(\pi, \psi)$ and every $g \in G$.

We will identify the functional $\mu$ on $\mathcal{W}(\pi, \psi)$ with the corresponding linear functional on $V$, which we will, by abuse of notation, also denote by $\mu$. By the uniqueness of the Whittaker model, this identification defines $\mu \in V^{*}$ in a unique way, up to a scalar multiple. Since $\mu \in\left(V^{*}\right)^{P_{n}(\mathbb{R})}$ and $\left(V^{*}\right)^{P_{n}(\mathbb{R})}=\left(V^{*}\right)^{G_{n}(\mathbb{R})}$ (see [Kemarsky 2015, Theorem 1.1]), we obtain that $\mu \in\left(V^{*}\right)^{G_{n}(\mathbb{R})}$. Clearly, $\mu \neq 0$. 
The functional $\mu$ defines an embedding of $V$ into the space of functions on $G_{n}(\mathbb{R}) \backslash G_{n}(\mathbb{C})$ via

$$
V \ni v \mapsto(g \mapsto \mu(\pi(g) v)) .
$$

By abuse of notation we denote this embedding again by $\mu$. Denote the image of the embedding $\mu$ by $\mathcal{C}_{G_{n}(\mathbb{R})}(\pi)$. In the other direction, we can define a map

$$
\theta: \mathcal{C}_{G_{n}(\mathbb{R})}(\pi) \rightarrow \mathcal{W}(\pi, \psi)
$$

by

$$
\theta: f \mapsto\left(g \mapsto \int_{U_{n}(\mathbb{R}) \backslash U_{n}(\mathbb{C})} f(u g) \psi^{-1}(u) d u\right)
$$

In this section we will prove that for every $n$ there exists an irreducible representation $(\pi, V)$ of $G_{n}(\mathbb{C})$ that is $G_{n}(\mathbb{R})$-distinguished and such that the integral (6-2) is absolutely convergent for every $K$-finite vector in $(\pi, V)$.

Suppose that we have a generic representation $(\pi, V) \in \operatorname{Irr}_{G_{n}(\mathbb{R})}\left(G_{n}(\mathbb{C})\right)$ and that the integral (6-2) is absolutely convergent for a $K$-finite function $f \in \mathcal{C}_{G_{n}(\mathbb{R})}(\pi)$. Then, from [Lapid and Mao 2014], the composition of maps $\theta(\mu(f))$ is equal to $c f$ for some constant $0 \neq c \in \mathbb{C}$.

Lemma 6.2. Let $(\pi, V) \in \operatorname{Irr}_{G_{n}(\mathbb{R})}\left(G_{n}(\mathbb{C})\right)$ be a generic representation. Suppose the integral

$$
\int_{U_{n}(\mathbb{R}) \backslash U_{n}(\mathbb{C})} W(u) \psi^{-1}(u) d u
$$

absolutely converges for every $K_{n}$-finite function $W \in \mathcal{W}(\pi, \psi)$. Then for every $W \in \mathcal{W}(\pi, \psi)$ there exists an $f \in \mathcal{C}_{G_{n}(\mathbb{R})}(\pi)$ such that

$$
W(g)=\int_{U_{n}(\mathbb{R}) \backslash U_{n}(\mathbb{C})} f(u g) \psi^{-1}(u) d u .
$$

Recall the decomposition (3-3):

$$
G_{n}(\mathbb{C})=G_{n}(\mathbb{R}) A^{+} K .
$$

The involution $g \rightarrow{ }^{t} g^{-1}$ preserves this decomposition. Let $\check{W}(g)=W\left({ }^{t} g^{-1}\right)$. The Whittaker model $\mathcal{W}\left(\tilde{\pi}, \psi^{-1}\right)$ of the contragredient representation of $(\pi, V)$ is given by

$$
\mathcal{W}\left(\tilde{\pi}, \psi^{-1}\right)=\{\check{W}: W \in \mathcal{W}(\pi, \psi)\} .
$$

If the conditions of Lemma 6.2 are satisfied for $\mathcal{W}(\pi, \psi)$ then they are also satisfied for the contragredient representation $\mathcal{W}\left(\tilde{\pi}, \psi^{-1}\right)$. Explicitly, if $W \in \mathcal{W}(\pi, \psi)$ 
is equal to

$$
W(g)=\int_{U_{n}(\mathbb{R}) \backslash U_{n}(\mathbb{C})} f(u g) \psi^{-1}(u) d u,
$$

then we have

$$
\check{W}(g)=\int_{U_{n}(\mathbb{R}) \backslash U_{n}(\mathbb{C})} \check{f}(u g) \psi(u) d u,
$$

where $\check{f}(g):=f\left({ }^{t} g^{-1}\right)$.

Lemma 6.3. Let $N>0$. Then there exists $a(\pi, V) \in \operatorname{Irr}_{G_{n}(\mathbb{R})}\left(G_{n}(\mathbb{C})\right)$ such that for every $K$-finite function $f \in V$ there is a constant $C>0$, depending only on $f$, that satisfies, for every $k \in K, a \in A, h \in G_{n}(\mathbb{R})$, the inequality

$$
|f(h a k)| \leq C(f)\|a\|_{H}^{-N} .
$$

Proof. By [Flensted-Jensen 1980], there exists a relatively discrete series $\mathcal{H}:=$ $L_{d s}^{2}\left(G_{n}(\mathbb{R}) \backslash G_{n}(\mathbb{C})\right)$. Moreover, every irreducible representation in $\mathcal{H}$ is isomorphic to some $I(\chi)$, where

$$
\chi(z)=\left((z /|z|)^{i_{1}},(z /|z|)^{i_{2}}, \ldots,(z /|z|)^{i_{n}}\right)
$$

and $i_{1}, \ldots, i_{n} \in \mathbb{Z}$. If $C>0$ is big enough and if $\left|i_{k}-i_{j}\right|>C>0$ for all $i \neq j$, then the $G_{n}(\mathbb{R}) \backslash G_{n}(\mathbb{C})$ model of the space $I(\chi)$ lies in $\mathcal{H}$, and the $(\mathfrak{g}, K)$-module generated by a $K$-finite function $0 \neq f_{\lambda} \in I(\chi)$ satisfies the properties of the lemma. Indeed, by [Flensted-Jensen 1980, p. 254] (see also [Kassel and Kobayashi 2013, Proposition 5.1]), if $C>0$ is big enough and if $\left|i_{k}-i_{j}\right|>C>0$ for all $j \neq k$, then $f_{\lambda}(h a k) \leq C^{\prime}\|a\|^{-N}$ for all $h \in H, a \in A^{+}$, and $k \in K$.

Clearly, $f_{\lambda}$ and right translations of $f_{\lambda}$ by $K$ satisfy the properties of our lemma. We should prove that the derivatives of $f_{\lambda}$ also satisfy similar growth properties. This is achieved by a classical idea, which is attributed to Harish-Chandra (see also an expository article by [Cowling et al. 1988]). The function $f_{\lambda}$ is $K$-finite, hence there exists a smooth function $e_{\alpha}$ of compact support such that $f_{\lambda} * e_{\alpha}=f_{\lambda}$. Thus, for $X \in \mathfrak{g}$ we have $d X\left(f_{\lambda}\right)=f_{\lambda} * d X\left(e_{\alpha}\right)$. It follows that the derivatives of $f_{\lambda}$ have the same decay properties that $f_{\lambda}$ has.

Finally, the $(\mathfrak{g}, K)$-module generated by $f_{\lambda}$ is of finite length. Consequently, it contains an irreducible admissible $(\mathfrak{g}, K)$-submodule satisfying the decay property (6-3).

If every $K$-finite function in ( $\pi, V)$ satisfies (6-3) we say that the representation $(\pi, V)$ decays faster than $N$. Note that if $(\pi, V)$ decays faster than $N$, then its contragredient $(\tilde{\pi}, \tilde{V})$ also decays faster than $N$. Indeed, we can realize $(\tilde{\pi}, \tilde{V})$ as $\tilde{V}=\{\check{f}: f \in V\}$, where $\check{f}(g):=f\left({ }^{t} g^{-1}\right)$. If $g=h a k$, then ${ }^{t} g^{-1}={ }^{t} h^{-1} a^{t} k^{-1}$. Hence, the property of fast decay is true for $\check{f}$ if and only if it is true for $f$. 
To obtain estimates of convergence of integrals over the unipotent matrices we need the next elementary result. Define $\Omega_{n}$ as the subset of all upper triangular unipotent matrices in $G_{n}(\mathbb{C})$ with $u_{i j}$ purely imaginary for $j>i$. Note that $\Omega_{n}$ is a fundamental domain for $U_{n}(\mathbb{R}) \backslash U_{n}(\mathbb{C})$.

Lemma 6.4. There exist $a C>0$ and $d>0$, which depend only on $n$, such that for every $u \in \Omega_{n}$ we have

$$
\|u\| \leq C\left\|u \bar{u}^{-1}\right\|^{d} .
$$

Proof. The proof is by induction on $n$. For $n=2$ it follows by direct computation: if $u=\left(\begin{array}{cc}1 & i x \\ 0 & 1\end{array}\right)$, then $u \bar{u}^{-1}=\left(\begin{array}{cc}1 & 2 i x \\ 0 & 1\end{array}\right)$ and the claim is satisfied.

For a general $n$, let us define $A_{j}$ to be the set of the entries in the $j$-th upper diagonal in $g$ :

$$
A_{0}=\left\{g_{11}, g_{22}, \ldots, g_{n n}\right\}, A_{1}=\left\{g_{12}, g_{23}, \ldots, g_{(n-1) n}\right\}, \ldots, A_{n-1}=\left\{g_{1 n}\right\} .
$$

Define $B_{j}:=\bigcup_{0 \leq i<j} A_{i}$. The crucial observation is that entry $(i, j)$ of $\bar{u}^{-1}$ with indices satisfying $j-i=k$ equals

$$
\bar{u}_{i j}^{-1}=u_{i j}+P_{i j}(u),
$$

where $P_{i j} \in \mathbb{C}\left[B_{k}\right]$ is a fixed polynomial which depends only on the entries $u_{l m}$ with indices $l-m<k$. Similarly,

$$
\left(u \bar{u}^{-1}\right)_{i j}=2 u_{i j}+Q_{i j}(u),
$$

where $Q_{i j} \in \mathbb{C}\left[B_{k}\right]$ is a fixed polynomial which depends only on the entries $u_{l m}$ with indices $l-m<k$. For example, let $n=3$. Then

$$
u=\left(\begin{array}{ccc}
1 & i x & i y \\
0 & 1 & i z \\
0 & 0 & 1
\end{array}\right), \bar{u}^{-1}=\left(\begin{array}{ccc}
1 & i x & i y-x z \\
0 & 1 & i z \\
0 & 0 & 1
\end{array}\right), u \bar{u}^{-1}=\left(\begin{array}{ccc}
1 & i x & 2 i y-2 x z \\
0 & 1 & i z \\
0 & 0 & 1
\end{array}\right) .
$$

Thus $P_{12}=P_{23}=0, P_{13}=-x z, Q_{12}=Q_{23}=0, Q_{13}=-2 x z$. Define $v=u \bar{u}^{-1}$ and define "partial seminorms" of $u$ by

$$
\|u\|_{k}=\sqrt{\sum_{(i, j): j-i \leq k}\left|u_{i j}\right|^{2}} .
$$

We will prove by induction on $k$, with base $k=1$, that for every $k$, there exist $C_{k}, d_{k}>0$ such that $\|u\|_{k}^{2} \leq C_{k}\|v\|_{k}^{d_{k}}$. As $\|u\|_{n}=\|u\|$, the result follows.

Let $k=1$. For $C_{1}=1, d_{1}=1$ we obtain the desired inequality. Suppose the claim is true for $k-1$; that is, suppose

$$
\|u\|_{k-1}^{2} \leq C_{k-1}\|v\|_{k-1}^{d_{k-1}} \text {. }
$$


We want to show a similar inequality for $k$. There exist $C, d>0$ such that for every $1 \leq i \leq n-k$ we have $\left|v_{i, i+k}\right| \geq\left|u_{i, i+k}\right|-C\|u\|_{k-1}^{d}$. For example, one can choose

$$
d=\max \left\{\operatorname{deg}\left(P_{i j}\right): i-j=k\right\}
$$

and a big enough constant $C$. Let $u$ be a given upper triangular unipotent matrix with purely imaginary entries above the diagonal. There exist constants $C^{\prime}, C^{\prime \prime}$ such that if for all $i$ we have $\left|u_{i, i+k}\right| \leq 2 C\|u\|_{k-1}^{d}$, then

$$
\|u\|_{k}^{2}=\|u\|_{k-1}^{2}+\sum_{i}\left|u_{i, i+k}\right|^{2} \leq C^{\prime}\|u\|_{k-1}^{2 d} \leq C^{\prime \prime}\|v\|_{k-1}^{2 d d_{k-1}} \leq C^{\prime \prime}\|v\|_{k}^{2 d d_{k-1}} .
$$

On the other hand, if for some $i$ we have $\left|u_{i, i+k}\right|>2 C\|u\|_{k-1}^{d}$, then we have the inequality $\left|v_{i, i+k}\right|>\left|u_{i, i+k}\right| / 2$ and there exists a constant $C^{\prime \prime \prime}$ such that

$$
\sum_{i=1}^{n-k}\left|u_{i, i+k}\right|^{2} \leq C^{\prime \prime \prime} \sum_{i=1}^{n-k}\left|v_{i, i+k}\right|^{2} \leq C^{\prime \prime \prime}\|v\|_{k} \leq C^{\prime \prime \prime}\|v\|_{k}^{2 d d_{k-1}} .
$$

Therefore, in both cases there are constants $C_{k}, d_{k}$ such that

$$
\|u\|_{k}^{2} \leq C_{k}\|v\|_{k}^{d_{k}} .
$$

Corollary 6.5. There exist a $C>0$ and $d>0$, which depend only on $n$, such that for every $u \in \Omega_{n}$ we have

$$
\|u\|_{H} \leq C\left\|u \bar{u}^{-1}\right\|_{H}^{d} .
$$

Proof. From Lemma 6.4 we know that there exist $C_{1}, d_{1}>0$ such that for every $u \in \Omega_{n}$ we have $\|u\|<C_{1}\left\|u \bar{u}^{-1}\right\|^{d_{1}}$. Similarly, one proves that there exist $C_{2}, d_{2}>0$ such that for every $u \in \Omega_{n}$ we have $\|u\|<C_{2}\left\|\bar{u} u^{-1}\right\|^{d_{2}}$. Define $C=\max \left\{C_{1}, C_{2}\right\}$, $d=\max \left\{d_{1}, d_{2}\right\}$. Then $\|u\|_{H} \leq C\left\|u \bar{u}^{-1}\right\|_{H}^{d}$ for every $u \in \Omega_{n}$.

Lemma 6.6. Let $N>0$ be sufficiently large. Then, for every irreducible, $G_{n}(\mathbb{R})$ distinguished representation $(\pi, V)$ of $G_{n}(\mathbb{C})$ with decay faster than $N$, the integral

$$
\int_{U_{n}(\mathbb{R}) \backslash U_{n}(\mathbb{C})} f(u g) d u
$$

absolutely converges for every $g \in G_{n}(\mathbb{C})$ and every $K$-finite function $f \in V$.

Proof. Let $(\pi, V)$ be a $G_{n}(\mathbb{R})$-distinguished representation of $G_{n}(\mathbb{C})$ such that for every $K$-finite function $f \in V$ there exists a $C>0$ depending only on $f$ such that

$$
|f(h a k)|<C\|a\|_{H}^{-N}
$$

for every $h \in G_{n}(\mathbb{R}), a \in A^{+}, k \in K$. Let $u g=h a k$. Then $(\overline{u g})^{-1} u g=\bar{g}^{-1}\left(\bar{u}^{-1} u\right) g$. Since $g$ is fixed, there exists a $C_{1}>0$ such that for every matrix $u \in G_{n}$ we have

$$
C_{1}^{-1}\left\|\bar{u}^{-1} u\right\|<\left\|(\overline{u g})^{-1} u g\right\|<C_{1}\left\|\bar{u}^{-1} u\right\| .
$$


By Lemma 6.4, for $u \in \Omega$ we have

$$
\|u\|<C_{2}\left\|\bar{u}^{-1} u\right\|^{d} .
$$

On the other hand,

$$
(\overline{u g})^{-1} u g=\bar{k}^{-1}\left(a \bar{a}^{-1}\right) k=\bar{k}^{-1} a^{2} k .
$$

Note that $k \in K$ is a unitary matrix, and therefore

$$
\left\|\bar{k}^{-1} a^{2} k\right\|=\left\|a^{2} k\right\|=\left\|a^{2}\right\| .
$$

Combining these inequalities we get

$$
\left\|a^{2}\right\|=\left\|(\overline{u g})^{-1} u g\right\|>C_{3}\left\|u \bar{u}^{-1}\right\|>C_{4}\|u\|^{1 / d} .
$$

Finally, we obtain that there exist constants $C, d^{\prime}$ such that for $u g=h a k$, where $u$ is in $\Omega_{n}$ and $g \in G_{n}$ is fixed, we have

$$
\|a\|>C\|u\|^{1 / d^{\prime}} .
$$

Therefore,

$$
\int_{U_{n}(\mathbb{R}) \backslash U_{n}(\mathbb{C})}|f(u g)| d u \leq \int_{\Omega} C\|u\|_{H}^{-N / d^{\prime}} d u .
$$

The integral in (6-4) converges for $N$ big enough, thus the lemma is proved.

Corollary 6.7. Let $N>0$ be sufficiently large. Then, for every irreducible $G_{n}(\mathbb{R})$ distinguished representation $(\pi, V)$ of $G_{n}(\mathbb{C})$ with decay faster than $N$, the integral (6-2) is absolutely convergent.

\section{Archimedean Asai integrals}

Nonarchimedean Asai integrals were introduced by Flicker [1988] who then used them to analyze the local and global Asai $L$ and $\epsilon$-factors [1993]. In this section we introduce an archimedean analog of Asai integrals and prove that they are of moderate growth. We also state a functional equation analogous to [Offen 2011, Lemma 4.2] that is satisfied by the integrals.

Let $(\pi, V)$ be a generic irreducible unitarizable representation of $G_{n}(\mathbb{C})$ and let $\mathcal{W}(\pi, \psi)$ be its Whittaker model. For $W \in \mathcal{W}(\pi, \psi)$, we define an archimedean Asai integral to be

$$
Z(s, W, \Phi)=\int_{U_{n}(\mathbb{R}) \backslash G_{n}(\mathbb{R})} W(g) \Phi((0,0, \ldots, 0,1) g)|\operatorname{det} g|_{\mathbb{R}}^{s} d g .
$$


Lemma 7.1. Let $\Phi \in \mathcal{S}\left(\mathbb{C}^{n}\right)$ and $\operatorname{Re}(s) \geq 1$. Then $W \mapsto Z(s, W$, $\Phi)$ defines a continuous functional on $\mathcal{W}(\pi, \psi)$ for $\operatorname{Re}(s) \geq 1$. That is, there exist a continuous seminorm $\mu$ on $\mathcal{W}(\pi, \psi)$ and a continuous seminorm $v$ on $\mathcal{S}\left(\mathbb{C}^{n}\right)$ such that

$$
|Z(s, W, \Phi)| \leq \mu(W) v(\Phi)
$$

for every $\Phi \in \mathcal{S}\left(\mathbb{C}^{n}\right)$ and every $W \in \mathcal{W}(\pi, \psi)$. As a consequence, there exist an $N>0$, a continuous seminorm $\mu^{\prime}$ on $\mathcal{W}(\pi, \psi)$ and a continuous seminorm $v$ on $\mathcal{S}\left(\mathbb{C}^{n}\right)$ such that

$$
\int_{U_{n}(\mathbb{R}) \backslash G_{n}(\mathbb{R})}\left|W^{\prime}(h g) \Phi((0,0, \ldots, 0,1) h g)\right||\operatorname{det} h|_{\mathbb{R}} d h \leq \mu^{\prime}(W) v^{\prime}(\Phi)\|g\|_{H}^{N}
$$

for every $g \in G$ and every $W^{\prime} \in \mathcal{W}(\pi, \psi)$.

Proof. Let $\delta_{0}$ be the modulus function of $B_{n}(\mathbb{R})$. Using the Iwasawa decomposition we obtain

$$
|Z(s, W, \Phi)| \leq \int_{K_{n}(\mathbb{R})} \int_{T_{n}}|W(t k) \Phi((0,0, \ldots, 0,1) t k)||\operatorname{det}(t)|_{\mathbb{R}}^{\operatorname{Re}(s)} \delta_{0}^{-1}(t) d t d k .
$$

By [Lapid and Mao 2014, Corollary 2.2] there exist a $\lambda>-1 / 2$, a $d>0$ and a continuous seminorm $\mu$ on $\mathcal{W}(\pi, \psi)$ such that

$$
|W(t k)| \leq \delta^{\frac{1}{2}}(t)|\operatorname{det} t|_{\mathbb{R}}^{2 \lambda}\left|t_{n}\right|_{\mathbb{R}}^{-2 n \lambda}(1+\|\log t\|)^{d} \mu(W)
$$

for every $t \in T_{n}$, every $k \in K_{n}$ and every $W \in \mathcal{W}(\pi, \psi)$. Note that

$$
\delta^{\frac{1}{2}}(t) \delta_{0}(t)^{-1}=1
$$

for all $t \in T_{n}$. The expression $\Phi((0,0, \ldots, 0,1) t k)=\Phi\left(\left(0,0, \ldots, 0, t_{n}\right) k\right)$ does not depend on $t_{1}, t_{2}, \ldots, t_{n-1}$. As in the proof of Lemma 5.2, we obtain for some $e>0$

$\begin{aligned}|Z(s, W, \Phi)| \leq \mu(W) & \prod_{j=1}^{n-1} \int_{1}^{\infty} t_{j}^{j(2 \lambda+\operatorname{Re}(s))}(1+\log t)_{j}^{e} d^{\times} t_{j} \\ & \times \int_{K_{n}(\mathbb{R})} \int_{0}^{\infty} t_{n}^{n R e(s)}\left(1+\left|\log t_{n}\right|\right)^{e} \Phi\left(\left(0,0, \ldots, 0, t_{n}\right) k\right) d^{\times} t_{n} d k .\end{aligned}$

Thus, $Z(s, W, \Phi)$ converges absolutely for $\operatorname{Re}(s)>\max (-2 \lambda, 0)$. Since $\lambda>-1 / 2$ for $\pi$ unitary and generic (see [Lapid and Mao 2014, p. 8]), the integral converges absolutely for $\operatorname{Re}(s) \geq 1$. For such $s$, there is a continuous seminorm $v$ on $\mathcal{S}\left(\mathbb{R}^{n}\right)$ 
such that

$$
\left|\int_{K_{n}(\mathbb{R})} \int_{0}^{\infty} t_{n}^{n R e(s)}\left(1+\left|\log t_{n}\right|\right)^{e} \Phi\left(\left(0,0, \ldots, 0, t_{n}\right) k\right) d^{\times} t_{n} d k\right| \leq v(\Phi)
$$

for every $\Phi \in \mathcal{S}\left(\mathbb{R}^{n}\right)$. As a consequence, there exist a continuous seminorm $\mu$ on $\mathcal{S}\left(\mathbb{R}^{n}\right)$ and a continuous seminorm $v$ on $\mathcal{W}(\pi, \psi)$ such that

$$
|Z(s, W, \Phi)| \leq \mu(\Phi) v(W)
$$

for every $\Phi \in \mathcal{S}\left(\mathbb{R}^{n}\right)$ and every $W \in \mathcal{W}(\pi, \psi)$. Thus there exist $M_{1}, M_{2}>0$ and continuous seminorms $\mu^{\prime}$ on $\mathcal{S}\left(\mathbb{R}^{n}\right)$ and $\nu^{\prime}$ on $\mathcal{W}(\pi, \psi)$ such that

$$
|Z(s, \pi(g) W, R(g) \Phi)| \leq \mu(R(g) \Phi) v(\pi(g) W) \leq\|g\|_{H}^{M_{1}}\|g\|_{H}^{M_{2}} \mu^{\prime}(\Phi) v^{\prime}(W)
$$

for every $g \in G$ and every $W \in \mathcal{W}(\pi, \psi)$.

The next lemma provides a functional equation for archimedean Asai integrals.

Lemma 7.2. Let $\pi$ be an irreducible, unitary, nondegenerate, $G_{n}(\mathbb{R})$-distinguished representation of $G_{n}(\mathbb{C})$. For every $\Phi \in \mathcal{S}\left(\mathbb{C}^{n}\right)$ and $W \in \mathcal{W}(\pi, \psi)$ we have

$$
Z\left(1, \tilde{W},\left.\hat{\Phi}\right|_{\mathbb{R}^{n}}\right)=c(\pi) Z\left(1, W,\left.\Phi\right|_{\mathbb{R}^{n}}\right) .
$$

Proof. For the proof see [Offen 2011, Lemma 4.2].

We will use the following technical result in the next section.

Lemma 7.3. Let $\left(\pi^{\prime}, V^{\prime}\right)$ be nondegenerate unitary representation of $G_{n}(\mathbb{C})$ and let $\mathcal{W}\left(\pi^{\prime}, \psi^{-1}\right)$ be its Whittaker model. Then there exists an $N>0$ such that for every irreducible, $G_{n}(\mathbb{R})$-distinguished representation $(\pi, V)$ of $G_{n}(\mathbb{C})$ with decay faster than $N$ and every function $f \in \mathcal{C}_{G_{n}}(\mathbb{R})(\pi)$, the following integral is absolutely convergent:

$\int_{G_{n}(\mathbb{R}) \backslash G_{n}(\mathbb{C})}|f(g)||\operatorname{det} g|_{\mathbb{C}}^{\frac{1}{2}}\left(\int_{U_{n}(\mathbb{R}) \backslash G_{n}(\mathbb{R})}\left|W^{\prime}(h g) \Phi((0,0, \ldots, 0,1) h g)\right||\operatorname{det} h|_{\mathbb{R}} d h\right) d g$.

Proof. This is an immediate consequence of Lemma 7.1.

\section{Equality of two functionals}

Let $(\pi, V) \in \operatorname{Irr}_{G_{n}(\mathbb{R})}\left(G_{n}(\mathbb{C})\right)$ be generic and unitarizable and let $\mathcal{W}(\pi, \psi)$ be its Whittaker model. Define linear functionals $\mu, \tilde{\mu} \in V^{*}$ on $\left.\mathcal{W}(\pi, \psi)\right)$ by

$$
\mu: W \mapsto \int_{U_{n-1}(\mathbb{R}) \backslash G_{n-1}(\mathbb{R})} W(g) d g \quad \text { and } \quad \tilde{\mu}: W \mapsto \int_{U_{n-1}(\mathbb{R}) \backslash G_{n-1}(\mathbb{R})} W\left(\left(\begin{array}{cc}
0 & 1 \\
I_{n-1} & 0
\end{array}\right)\left(\begin{array}{ll}
g & 0 \\
0 & 1
\end{array}\right)\right) d g \text {. }
$$


Since $\mu, \tilde{\mu} \in\left(V^{*}\right)^{P_{n}(\mathbb{R})}$ and $\left(V^{*}\right)^{P_{n}(\mathbb{R})}=\left(V^{*}\right)^{G_{n}(\mathbb{R})}$ (see [Kemarsky 2015, Theorem 1.1]), we obtain that $\mu, \tilde{\mu} \in\left(V^{*}\right)^{G_{n}(\mathbb{R})}$. Clearly, the functionals $\mu, \tilde{\mu}$ are nonzero. The space of $G_{n}(\mathbb{R})$-invariant continuous functionals on $V$ is one-dimensional (see [Aizenbud and Gourevitch 2009, Theorem 8.2.5]), thus there exists a proportionality constant $c(\pi) \neq 0$ such that $\tilde{\mu}=c(\pi) \mu$.

The goal of this section is to calculate the proportionality factor $c(\pi)$ by proving the following theorem.

Theorem 8.1. Let $(\pi, V) \in \operatorname{Irr}_{G_{n}(\mathbb{R})}\left(G_{n}(\mathbb{C})\right)$. Then $c(\pi)=1$.

We now state an archimedean analogue of [Offen 2011, Lemma 6.1].

Lemma 8.2. Let $\pi^{\prime} \in \operatorname{Irr}_{G_{n}(\mathbb{R})}\left(G_{n}(\mathbb{C})\right)$ be generic and unitarizable. Then there exists a generic and unitarizable $\pi \in \operatorname{Irr}_{G_{n}(\mathbb{R})}\left(G_{n}(\mathbb{C})\right)$ such that

$$
\gamma\left(\frac{1}{2}, \pi \times \pi^{\prime} ; \psi\right)=c\left(\pi^{\prime}\right) .
$$

Note that for $\pi, \pi^{\prime}$ as in Lemma 8.2 we already know that $\gamma\left(1 / 2, \pi \times \pi^{\prime}, \psi\right)=1$. As a result, the equality $c\left(\pi^{\prime}\right)=1$ follows.

The proof of Lemma 8.2 is similar to the proof of [Offen 2011, Lemma 6.1]. However, in the archimedean case, there are convergence issues that we need to check.

Proof of Lemma 8.2. Let $W \in \mathcal{W}(\pi, \psi), W^{\prime} \in \mathcal{W}\left(\pi^{\prime}, \psi^{-1}\right)$, and $\Phi \in \mathcal{S}\left(\mathbb{C}^{n}\right)$. The idea is to prove an equality of Rankin-Selberg integrals of the type

$$
\Psi\left(\frac{1}{2}, \tilde{W}, \tilde{W}^{\prime} ; \hat{\Phi}\right)=c\left(\pi^{\prime}\right) \Psi\left(\frac{1}{2}, W, W^{\prime} ; \Phi\right) .
$$

Actually, it is enough to prove such an equality for a pair of functions $W \in \mathcal{W}(\pi, \psi)$ and $W^{\prime} \in \mathcal{W}\left(\pi^{\prime}, \psi\right)$ such that at least one of the integrals $\Psi\left(1 / 2, \tilde{W}, \tilde{W}^{\prime} ; \hat{\Phi}\right)$, $\Psi\left(1 / 2, W, W^{\prime} ; \Phi\right)$ is nonzero (and thus both integrals are nonzero).

We will obtain the necessary convergence estimates for every $K_{n}$-finite function $W \in \mathcal{W}(\pi, \psi)$ and every function $W^{\prime} \in \mathcal{W}\left(\pi^{\prime}, \psi\right)$. By our classification of $G_{n}(\mathbb{R})$ distinguished representations of $G_{n}(\mathbb{C})$, the central character $\omega_{\pi}$ of the $G_{n}(\mathbb{R})$ distinguished representation satisfies $\omega_{\pi}(-1)=1$. Thus, by Theorem 5.1, we have the equality

$$
\Psi\left(1-s, \tilde{W}, \tilde{W}^{\prime} ; \hat{\Phi}\right)=\gamma\left(s, \pi \times \pi^{\prime}, \psi\right) \Psi\left(s, W, W^{\prime} ; \Phi\right) .
$$

Let $f \in \mathcal{C}_{G_{n}(\mathbb{R})}(\pi)$ be such that

$$
W(g)=\int_{U_{n}(\mathbb{R}) \backslash U_{n}(\mathbb{C})} f(u g) \psi^{-1}(u) d u .
$$


We will prove the absolute convergence of the following integrals at $s=1 / 2$ :

$$
\begin{aligned}
\int_{U_{n}(\mathbb{C}) \backslash G_{n}(\mathbb{C})}\left|W(g) W^{\prime}(g) \Phi((0,0, \ldots, 0,1) g)\right||\operatorname{det} g|_{\mathbb{C}}^{s} d g \\
\leq \int_{U_{n}(\mathbb{C}) \backslash G_{n}(\mathbb{C})}\left(\int_{U_{n}(\mathbb{R}) \backslash U_{n}(\mathbb{C})}|f(u g)| d u\right) \\
=\int_{U_{n}(\mathbb{C}) \backslash G_{n}(\mathbb{C})}\left|f(g) W^{\prime}(g) \Phi((0,0, \ldots, 0,1) g)\right||\operatorname{det} g|_{\mathbb{C}}^{s} d g \\
=\int_{G_{n}(\mathbb{R}) \backslash W_{n}(\mathbb{C})}|f(g)||\operatorname{det} g|_{\mathbb{C}}^{s} \\
\left.\quad \times \int_{U_{n}(\mathbb{R}) \backslash G_{n}(\mathbb{R})}\left|W^{\prime}(h g) \Phi((0,0, \ldots, 0,1) h g)\right||\operatorname{det} h|_{\mathbb{R}}^{2 s} d h\right) d g .
\end{aligned}
$$

The left-hand side of (8-2) is absolutely convergent by Lemma 5.2 and the integrals on the right-hand side of (8-2) are absolutely convergent by Lemmas 7.1 and 7.3. Using absolute convergence for $s=1 / 2$ of the integrals appearing in (8-2) we obtain the equality (8-1) by the following argument:

$$
\begin{aligned}
\Psi & \left(\frac{1}{2}, W, W^{\prime} ; \Phi\right) \\
= & \int_{U_{n}(\mathbb{C}) \backslash G_{n}(\mathbb{C})} W(g) W^{\prime}(g) \Phi((0,0, \ldots, 0,1) g)|\operatorname{det} g|_{\mathbb{C}}^{\frac{1}{2}} d g \\
= & \int_{U_{n}(\mathbb{C}) \backslash G_{n}(\mathbb{C})}\left(\int_{U_{n}(\mathbb{R}) \backslash U_{n}(\mathbb{C})} f(u g) \psi_{n}^{-1}(u) d u\right) W^{\prime}(g) \Phi((0,0, \ldots, 0,1) g)|\operatorname{det} g|_{\mathbb{C}}^{\frac{1}{2}} d g \\
= & \int_{U_{n}(\mathbb{C}) \backslash G_{n}(\mathbb{C})} f(g) W^{\prime}(g) \Phi((0,0, \ldots, 0,1) g)|\operatorname{det} g|_{\mathbb{C}}^{\frac{1}{2}} d g \\
= & \int_{G_{n}(\mathbb{R}) \backslash G_{n}(\mathbb{C})} f(g)|\operatorname{det} g|_{\mathbb{C}}^{\frac{1}{2}}\left(\int_{U_{n}(\mathbb{R}) \backslash G_{n}(\mathbb{R})} W^{\prime}(h g) \Phi((0,0, \ldots, 0,1) h g)|\operatorname{det} h|_{\mathbb{R}} d h\right) d g \\
= & \int_{G_{n}(\mathbb{R}) \backslash G_{n}(\mathbb{C})} f(g)|\operatorname{det} g|_{\mathbb{C}}^{\frac{1}{2}} Z\left(1, \pi^{\prime}(g) W^{\prime},\left.\Phi(\cdot g)\right|_{\left.\mathbb{R}^{n}\right) d g .}\right.
\end{aligned}
$$

Define $f^{*}(g):=f\left({ }^{t} g^{-1}\right)$. Then, clearly, $f^{*} \in \mathcal{C}_{G_{n}(\mathbb{R})}(\tilde{\pi})$. Applying the change of variables $u \rightarrow w_{n}{ }^{t} u^{-1} w_{n}^{-1}$ and the fact that $f\left(w_{n} g\right)=f(g)$, it follows from the 
definitions that

$$
\tilde{W}(g)=\int_{U_{n}(\mathbb{R}) \backslash U_{n}(\mathbb{C})} f^{*}(u g) \psi(u) d u .
$$

The same computation applied to $\tilde{\pi}$ and $\tilde{\pi}^{\prime}$ yields

$$
\Psi\left(\frac{1}{2}, \tilde{W}, \tilde{W}^{\prime} ; \hat{\Phi}\right)=\int_{G_{n}(\mathbb{R}) \backslash G_{n}(\mathbb{C})} f^{*}(g)|\operatorname{det} g|_{\mathbb{C}}^{\frac{1}{2}} Z\left(1, \tilde{\pi}^{\prime}(g) \tilde{W}^{\prime},\left.\hat{\Phi}(\cdot g)\right|_{\mathbb{R}^{n}}\right) d g .
$$

By Lemma 7.2,

$$
Z\left(1, \tilde{\pi}^{\prime}\left({ }^{t} g^{-1}\right) \tilde{W}^{\prime},\left.\hat{\Phi}\left(\cdot{ }^{t} g^{-1}\right)\right|_{\mathbb{R}^{n}}\right)=c\left(\pi^{\prime}\right)|\operatorname{det} g|_{\mathbb{C}} Z\left(1, \pi^{\prime}(g) W^{\prime},\left.\Phi(\cdot g)\right|_{\mathbb{R}^{n}}\right) .
$$

Finally, we obtain

$$
\Psi\left(\frac{1}{2}, \tilde{W}, \tilde{W}^{\prime} ; \hat{\Phi}\right)=c\left(\pi^{\prime}\right) \Psi\left(\frac{1}{2}, W, W^{\prime} ; \Phi\right)
$$

for every $K_{n}$-finite function $W \in \mathcal{W}(\pi, \psi), W^{\prime} \in \mathcal{W}\left(\pi^{\prime}, \psi^{-1}\right)$ and every $\Phi \in \mathcal{S}\left(\mathbb{C}^{n}\right)$. It is well-known that there exist $K_{n}$-finite $W \in \mathcal{W}(\pi, \psi), W^{\prime} \in \mathcal{W}\left(\pi^{\prime}, \psi^{-1}\right)$ such that $\Psi\left(1 / 2, W, W^{\prime} ; \Phi\right) \neq 0$. It follows that $c\left(\pi^{\prime}\right)=\gamma\left(1 / 2, \pi \times \pi^{\prime} ; \psi\right)$.

\section{Appendix A: Generic Langlands quotient}

In this section we sketch a proof of the well-known fact that the Langlands quotient of $I(\chi)$ is generic if and only if $I(\chi)$ is irreducible. This fact follows from the papers of Kostant [1978] and Vogan [1978]. For the convenience of the reader we rewrite it here. Similar results for $\mathrm{GL}_{n}(\mathbb{R})$ were obtained by Casselman and Zuckerman.

Let $\mathfrak{g}=M_{n}(\mathbb{C})$ be the Lie algebra of $G_{n}(\mathbb{C})$ and let $K$ be the standard maximal compact subgroup of $G_{n}(\mathbb{C})$.

Definition. An irreducible ( $\mathfrak{g}, K)$-module $X$ is called large if its annihilator in the universal enveloping algebra $U(\mathfrak{g})$ is a minimal primitive ideal. We will say that a smooth irreducible representation $(\pi, V)$ of $G_{n}(\mathbb{C})$ is large if the corresponding $(\mathfrak{g}, K)$-module consisting of $K$-finite vectors in $V$ is large.

Let $\chi=\left(\chi_{1}, \chi_{2}, \ldots, \chi_{n}\right)$ be a character of $B_{n}(\mathbb{C})$ and suppose $\left|\chi_{j}(t)\right|=|t|^{\lambda_{j}}$ with $\lambda_{1} \geq \lambda_{2} \geq \cdots \geq \lambda_{n}$. By [Vogan 1978, Theorem 6.2], if $(\sigma, W)$ is an irreducible subrepresentation of $I(\chi)$ then $(\sigma, W)$ is large. Suppose $(\pi, V)$ is the Langlands quotient of $I(\chi)$ and suppose $(\pi, V)$ is generic. Then by Kostant's theorem $(\pi, V)$ is large. On the other hand, [Vogan 1978, Corollary 6.7] states that there is a unique large composition factor in the composition series for $I(\chi)$. We obtain $(\pi, V) \simeq(\sigma, W)$ and thus $(\sigma, W)=I(\chi)$; that is, $I(\chi)$ is an irreducible representation. 


\section{Appendix B: Gamma factors: converse direction}

Fix a smooth, irreducible, generic and admissible representation $(\pi, V)$ of $G_{n}(\mathbb{C})$. Suppose we know that

$$
\gamma\left(\frac{1}{2}, \pi \times \pi^{\prime}, \psi\right)=1
$$

for every $m \leq k$ and every smooth irreducible $G_{m}(\mathbb{R})$-distinguished representation $\left(\pi^{\prime}, V^{\prime}\right)$ of $G_{m}(\mathbb{C})$. What is the minimal $k$ such that (B-1) implies that $(\pi, V)$ is $G_{n}(\mathbb{R})$-distinguished? In this section we give an answer to this question in the case when $(\pi, V)$ is a unitary representation.

In the following two theorems we prove that $k=1$ is enough. Theorem B. 1 is a particular case of Theorem B.2. Nevertheless we state and prove it since the proof of Theorem B.1 is simpler than and may aid in the understanding of the proof of Theorem B.2.

Theorem B.1. Let $\chi=\left(\chi_{1}, \chi_{2}, \ldots, \chi_{n}\right)$ be a unitary character of $B_{n}$ and suppose that $\chi_{j}(z)=|z|_{\mathbb{C}}^{s_{j}}(z /|z|)^{k_{j}}$ with $s_{j}$ purely imaginary and $k_{j} \in \mathbb{Z}$ for every $1 \leq j \leq n$. Suppose $(\pi, V)=I(\chi)$ is a smooth, generic and irreducible representation of $G_{n}(\mathbb{C})$. Finally, suppose

$$
\gamma\left(\frac{1}{2}, \pi \times \chi^{\prime}, \psi\right)=1
$$

for every $\mathbb{R}^{\times}$-distinguished unitary character $\chi^{\prime}: \mathbb{C}^{\times} \rightarrow \mathbb{R}^{\times}$. Then there exists an involution $w \in S_{n}$ such that $w \chi=\overline{\left(\chi^{-1}\right)}$. Moreover, one can find an involution $w \in S_{n}$ such that $w \chi=\overline{\left(\chi^{-1}\right)}$ and such that, for every fixed point $w(i)=i$, the integer $k_{i}$ is even.

Proof. Observe that every $\mathbb{R}^{\times}$-distinguished unitary character $\chi^{\prime}: \mathbb{C} \rightarrow \mathbb{R}^{\times}$is of the form $\chi(z)=(z /|z|)^{2 m}$ for $m \in \mathbb{Z}$. By [Jacquet 2009, Lemma 16.3] we have

$$
\gamma\left(\frac{1}{2}, \operatorname{Ind}(\chi) \times \chi^{\prime}, \psi\right)=\prod_{i=1}^{n} \gamma\left(\frac{1}{2}, \chi_{i} \chi^{\prime}, \psi\right),
$$

where $\gamma\left(1 / 2, \chi_{i} \chi^{\prime}, \psi\right)$ is the one-dimensional Tate gamma factor. Following Tate [Cassels and Frölich 1967], denote $c_{m}(z)=(z /|z|)^{m}$ and recall that the Tate gamma factor is given by

where

$$
\gamma\left(s, c_{m}, \psi\right)=\epsilon_{m} \frac{(2 \pi)^{1-s} \Gamma\left(s+\frac{|m|}{2}\right)}{(2 \pi)^{s} \Gamma\left((1-s)+\frac{|m|}{2}\right)},
$$

$$
\epsilon_{m}= \begin{cases}1 & \text { if } m \text { is even or } m>0, \\ -1 & \text { if } m \text { is odd and } m<0\end{cases}
$$


Let us rewrite the equality $\gamma\left(1 / 2, \operatorname{Ind}(\chi) \times \chi^{\prime}, \psi\right)=1$ as

$$
\prod_{i=1}^{n} \epsilon_{2 m+k_{i}} \frac{(2 \pi)^{\frac{1}{2}-s_{i}}}{(2 \pi)^{\frac{1}{2}+s_{i}}} \frac{\Gamma\left(\frac{1}{2}+s_{i}+\frac{\left|k_{i}+2 m\right|}{2}\right)}{\Gamma\left(\frac{1}{2}-s_{i}+\frac{\left|k_{i}+2 m\right|}{2}\right)}=1
$$

for every $m \in \mathbb{Z}$. The product in (B-2) breaks into three products:

$$
\begin{aligned}
& p_{m, 1}=\prod_{i=1}^{n} \epsilon_{2 m+k_{i}}, \\
& p_{m, 2}=\prod_{i=1}^{n} \frac{(2 \pi)^{\frac{1}{2}-s_{i}}}{(2 \pi)^{\frac{1}{2}+s_{i}}=(2 \pi)^{-2 s_{1}-2 s_{2}-\cdots-2 s_{n}},} \\
& p_{m, 3}=\prod_{i=1}^{n} \frac{\Gamma\left(\frac{1}{2}+s_{i}+\frac{\left|k_{i}+2 m\right|}{2}\right)}{\Gamma\left(\frac{1}{2}-s_{i}+\frac{\left|k_{i}+2 m\right|}{2}\right)} .
\end{aligned}
$$

Note that the term $p_{m, 2}$ is constant (does not depend on $m$ ) and the term $p_{m, 1}$ stabilizes (that is, $p_{m, 1}=p_{m+1,1}$ for large enough and for small enough $m$ ). Also, we have $\left|k_{i}+m\right|=k_{i}+m$ for $m$ large enough. Let us take $m$ large enough and look at the expression

$$
\frac{p_{m+1,1} p_{m+1,2} p_{m+1,3}}{p_{m, 1} p_{m, 2} p_{m, 3}} .
$$

By our assumption this fraction equals 1 for every $m$. For $m$ large enough we have $p_{m+1,1} p_{m+1,2}=p_{m, 1} p_{m, 2}$, so $p_{m+1,3} / p_{m, 3}=1$. By the functional equation $\Gamma(z+1)=z \Gamma(z)$ we obtain

$$
1=\frac{p_{m+1,3}}{p_{m, 3}}=\prod_{i=1}^{n} \frac{\left(\frac{1}{2}+s_{i}+\frac{k_{i}+2 m}{2}\right)}{\left(\frac{1}{2}-s_{i}+\frac{k_{i}+2 m}{2}\right)} .
$$

Thus,

$$
\prod_{i=1}^{n}\left(\frac{1}{2}+s_{i}+\frac{k_{i}+2 m}{2}\right)=\prod_{i=1}^{n}\left(\frac{1}{2}-s_{i}+\frac{k_{i}+2 m}{2}\right)
$$

for large enough $m \in \mathbb{Z}$. Since both sides are polynomials in $m$, the polynomials are equal. As a consequence, the zeros of these two polynomials coincide; that is, for every $1 \leq i \leq n$ there exists a $1 \leq j \leq n$ such that

$$
\frac{1}{2}-s_{i}+\frac{k_{i}}{2}=\frac{1}{2}+s_{j}+\frac{k_{j}}{2} .
$$

By our assumption the $s_{i} \mathrm{~s}$ are purely imaginary and the $k_{i} \mathrm{~s}$ are integers. Thus, $-s_{i}=s_{j}$ and $k_{i}=k_{j}$. Note that $\bar{s}_{i}=-s_{i}$ and this means exactly that we can define $w(i)=j, w(j)=i$ and $\chi_{j}=\bar{\chi}_{i}^{-1}=\chi_{w(i)}$. Therefore, there exists an involution $w \in S_{n}$ such that $w(\chi)=\bar{\chi}^{-1}$. 
From the proof of the existence of an involution $w$ it follows that $\sum_{j=1}^{n} s_{j}=0$ and that the products $p_{m, 2}=1$ and $p_{m, 3}=1$ for every $m \in \mathbb{Z}$. This establishes the existence of an involution $w \in S_{n}$ such that $w(\chi)=\bar{\chi}^{-1}$. It remains to establish the second property: existence of an involution such that, in addition, for every fixed point $w(j)=j$ of the involution the corresponding integer $k_{j}$ is even. Note that if $i$ is a fixed point of $w$ then $s_{i}=0$. Without loss of generality assume that if $w(i)=i$ and $w(j)=j$, then $k_{i} \neq k_{j}$. Otherwise we can define an involution $w^{\prime}$ by $w^{\prime}(i)=j, w^{\prime}(j)=i$ and $w^{\prime}(l)=w(l)$ for $l \neq i, j$ and the new involution $w^{\prime}$ also satisfies $w^{\prime}(\chi)=\bar{\chi}^{-1}$.

Assume on the contrary that $w(i)=i$ but that $k_{i}$ is odd. Then take two consecutive products $p_{m, 1}$ and $p_{m+1,1}$ for $m=\left(-k_{i}-1\right) / 2$. Observe that $\epsilon_{2 m+k_{i}}=-\epsilon_{2(m+1)+k_{i}}$ and that the other terms appearing in the products $p_{m, 1}$ and $p_{m+1,1}$ equal each other respectively. As a consequence, $p_{m+1,1}=-p_{m, 1}$. But from the preceding paragraph we have $p_{m, 2}=p_{m+1,2}=1$ and also $p_{m, 3}=p_{m+1,3}=1$ and thus $p_{m+1,1}=p_{m, 1}=1$. Contradiction!

Therefore, if $w(i)=i$ then the integer $k_{i}$ is even; that is, $\chi_{i}(-1)=1$.

A small modification of this proof gives a stronger theorem.

Theorem B.2. Let $\chi=\left(\chi_{1}, \chi_{2}, \ldots, \chi_{n}\right)$ be a character of $B_{n}$ and suppose that $\chi_{j}(z)=|z|_{\mathbb{C}}^{s_{j}}(z /|z|)^{k_{j}}$ with $-1 / 2<\operatorname{Re}\left(s_{j}\right)<1 / 2$ and $k_{j} \in \mathbb{Z}$ for every $1 \leq j \leq n$. Suppose $(\pi, V)=I(\chi)$ is a smooth, generic, irreducible representation of $G_{n}(\mathbb{C})$. Finally, suppose

$$
\gamma\left(\frac{1}{2}, \pi \times \chi^{\prime}, \psi\right)=1
$$

for every $\mathbb{R}^{\times}$-distinguished unitary character $\chi^{\prime}: \mathbb{C}^{\times} \rightarrow \mathbb{R}^{\times}$. Then there exists an involution $w \in S_{n}$ such that $w \chi=\overline{\left(\chi^{-1}\right)}$. Moreover, one can find an involution $w \in S_{n}$ such that $w \chi=\overline{\left(\chi^{-1}\right)}$ and such that, for every fixed point $w(i)=i$, the integer $k_{i}$ is even.

Proof. By the same argument as in the previous theorem we obtain that for every $1 \leq i \leq n$ there exists a $1 \leq j \leq n$ such that

$$
\frac{1}{2}-s_{i}+\frac{k_{i}}{2}=\frac{1}{2}+s_{j}+\frac{k_{j}}{2} .
$$

By subtracting $1 / 2$ from both sides of this equality and taking real parts we can replace $s_{j}$ by $\operatorname{Re}\left(s_{j}\right)$. Thus we can assume that for every $1 \leq i \leq n$ we have $-1 / 2<s_{i}<1 / 2$ and also for every $1 \leq i \leq n$ there exists a $1 \leq j \leq n$ such that $-s_{i}+k_{i} / 2=s_{j}+k_{j} / 2$. Multiply both sides of this equation by 2 and replace $s_{i}$ by $2 s_{i}$. Then, we can assume that for every $1 \leq i \leq n$ we have $-1<s_{i}<1$ and also for every $1 \leq i \leq n$ there exists a $1 \leq j \leq n$ such that

$$
-s_{i}+k_{i}=s_{j}+k_{j}
$$


Let us call this condition the "antisymmetry condition". The claim is that the "antisymmetry condition" implies that there exists an involution $w \in S_{n}$ such that $w(\chi)=\bar{\chi}^{-1}$; that is, if $w(i)=j$ then $s_{i}=-s_{j}$ and $k_{i}=k_{j}$. The proof of the existence of an involution $w$ is by induction on $n$. Clearly, for $n=1$ the condition $-s_{1}+k_{1}=s_{1}+k_{1}$ gives us $s_{1}=0$ and thus the identity involution $w(1)=1$ works. For a general $n$ it is enough that the "antisymmetry condition" implies that there is a pair $i, j$ such that $s_{i}=-s_{j}$ and $k_{i}=k_{j}$. Note that if $i=j$ then $s_{i}=-s_{i}$ implies $s_{i}=0$.

Suppose on the contrary that there are $\left\{s_{i}\right\}_{i=1}^{n} \subset(-1,1)$ and $\left\{k_{i}\right\}_{i=1}^{n} \subset \mathbb{Z}$ that satisfy the "antisymmetry condition", but there is no pair of indices $1 \leq i, j \leq n$ that satisfy $s_{i}=-s_{j}$ and $k_{i}=k_{j}$. In particular, there is some $1 \leq i \leq n$ such that $-s_{1}+k_{1}=s_{i}+k_{i}$. By our assumption we have $i>1$, so without loss of generality assume $i=2$. Let us assume $s_{1}>0$; the proof in the case $s_{1}<0$ is similar and $s_{1}=0$ is not possible by our assumption. We obtain $k_{1}-k_{2}=s_{1}+s_{2}$. The left-hand side is an integer and we have $-1<s_{1}+s_{2}<2$. Thus $s_{1}+s_{2}=0$ or $s_{1}+s_{2}=1$. The case $s_{1}+s_{2}=0$ is not possible by our assumption, thus $s_{1}+s_{2}=1$ and as a corollary $s_{2}>0$ and $k_{2}=k_{1}-1$. Similarly, there is some $1 \leq i \leq n$ such that $-s_{2}+k_{2}=s_{i}+k_{i}$. By the same argument we obtain $s_{i}>0$ and $k_{i}=k_{2}-1$. Thus $i \neq 1,2$ and without loss of generality we can assume $i=3$. Continuing in this manner we obtain an infinite sequence of integers $k_{j}$ such that $k_{j}=k_{1}+(j-1)$. Contradiction!

Thus there is a pair of indices $1 \leq i, j \leq n$ such that $s_{i}=-s_{j}$ and $k_{i}=k_{j}$. Removing them from our sequence of length $n$ we obtain a shorter sequence which satisfies the "antisymmetry condition".

Thus, we have proved that there is an involution $w \in S_{n}$ such that $w(\chi)=\bar{\chi}^{-1}$. The rest of the argument, that is, the proof of the existence of an involution $w$ such that for every fixed point $j$ of the involution the corresponding integer $k_{j}$ is even, is the same as in the proof of the previous theorem.

As a corollary, using the Tadic-Vogan classification of the unitary dual of $G_{n}(\mathbb{C})$, we obtain the following theorem.

Theorem B.3. Let $\chi=\left(\chi_{1}, \chi_{2}, \ldots, \chi_{n}\right)$ be a character of $B_{n}$ and suppose that $(\pi, V)=\operatorname{Ind}(\chi)$ is a smooth, generic, irreducible, and unitary representation of $G_{n}(\mathbb{C})$. Suppose

$$
\gamma\left(\frac{1}{2}, \pi \times \chi^{\prime}, \psi\right)=1
$$

for every $\mathbb{R}^{\times}$-distinguished unitary character $\chi^{\prime}: \mathbb{C}^{\times} \rightarrow \mathbb{R}^{\times}$. Then there exists an involution $w \in S_{n}$ such that $w \chi=\overline{\left(\chi^{-1}\right)}$. Moreover, one can find an involution $w \in S_{n}$ such that $w \chi=\overline{\left(\chi^{-1}\right)}$ and such that, for every fixed point $w(i)=i$, the integer $k_{i}$ is even. 
Proof. Let us define $\chi_{j}(z)=|z|_{\mathbb{C}}^{s_{j}}(z /|z|)^{k_{j}}$, where $s_{j} \in \mathbb{C}$ and $k_{j} \in \mathbb{Z}$. The theorem follows from Theorem B.2 and the fact that the unitaricity of $\operatorname{Ind}(\chi)$ implies $-1 / 2<\operatorname{Re}\left(s_{j}\right)<1 / 2$ for every $1 \leq j \leq n$ (see [Tadić 1985, Theorem A]).

Finally, by [Panichi 2001, Theorem 3.3.6] we know that an irreducible tempered representation $(\pi, V)$ of $G_{n}(\mathbb{C})$ is $G_{n}(\mathbb{R})$-distinguished if and only if there exists an involution $w \in S_{n}$ such that $w \chi=\overline{\left(\chi^{-1}\right)}$ and such that, for every fixed point $w(i)=i$, the integer $k_{i}$ is even. Therefore, an irreducible tempered representation $(\pi, V)$ of $G_{n}(\mathbb{C})$ is $G_{n}(\mathbb{R})$-distinguished if and only if

$$
\gamma\left(\frac{1}{2}, \pi \times \chi^{\prime}, \psi\right)=1
$$

for every $\mathbb{R}^{\times}$-distinguished unitary character $\chi^{\prime}: \mathbb{C}^{\times} \rightarrow \mathbb{R}^{\times}$.

\section{Acknowledgements}

I would like to thank Omer Offen for posing to me this question and providing many explanations on the subject.

I am grateful to Dmitry Gourevitch and Erez Lapid for many fruitful discussions and their help.

During the conference in Jussieu, June 2014, I told the results of this paper to Herve Jacquet. I would like to thank him, as his comments were very helpful.

I also wish to thank Avraham Aizenbud, Uri Bader, Max Gurevich, Job Kuit, Nadir Matringe, Amos Nevo, Henrik Schlictkrull and Dror Speiser for useful conversations and remarks.

\section{References}

[Aizenbud and Gourevitch 2009] A. Aizenbud and D. Gourevitch, "Generalized Harish-Chandra descent, Gelfand pairs, and an Archimedean analog of Jacquet-Rallis's theorem", Duke Math. J. 149:3 (2009), 509-567. MR 2011c:22026 Zbl 1221.22018

[Aizenbud and Lapid 2012] A. Aizenbud and E. Lapid, "Distinguished representations in the Archimedean case", 2012. Appendix D to B. Feigon, E. Lapid, and O. Offen, "On representations distinguished by unitary groups”, Publ. Math. Inst. Hautes Études Sci. 115 (2012), 185-323. MR 2930996 Zbl 06112182

[Aizenbud et al. 2008] A. Aizenbud, D. Gourevitch, and E. Sayag, " $\left(\mathrm{GL}_{n+1}(F), \mathrm{GL}_{n}(F)\right)$ is a Gelfand pair for any local field F”, Compos. Math. 144:6 (2008), 1504-1524. MR 2009k:22022 Zbl 1157.22004

[Cassels and Frölich 1967] J. W. S. Cassels and A. Frölich, Algebraic number theory, Academic Press, London, 1967. MR 35 \#6500 Zbl 0153.07403

[Cowling et al. 1988] M. Cowling, U. Haagerup, and R. Howe, "Almost $L^{2}$ matrix coefficients", J. Reine Angew. Math. 387 (1988), 97-110. MR 89i:22008 Zbl 0638.22004

[Flensted-Jensen 1980] M. Flensted-Jensen, "Discrete series for semisimple symmetric spaces", Ann. of Math. (2) 111:2 (1980), 253-311. MR 81h:22015 Zbl 0462.22006 
[Flicker 1988] Y. Z. Flicker, “Twisted tensors and Euler products”, Bull. Soc. Math. France 116:3 (1988), 295-313. MR 89m:11049 Zbl 0674.10026

[Flicker 1993] Y. Z. Flicker, "On zeroes of the twisted tensor L-function”, Math. Ann. 297:2 (1993), 199-219. MR 95c:11065 Zbl 0786.11030

[Jacquet 2009] H. Jacquet, "Archimedean Rankin-Selberg integrals", pp. 57-172 in Automorphic forms and L-functions II: Local aspects, edited by D. Ginzburg et al., Contemp. Math. 489, Amer. Math. Soc., Providence, RI, 2009. MR 2011a:11103 Zbl 1250.11051

[Kassel and Kobayashi 2013] F. Kassel and T. Kobayashi, "Poincaré series for non-Riemannian locally symmetric spaces”, preprint, 2013. arXiv 1209.4075

[Kemarsky 2015] A. Kemarsky, "Distinguished representations of $G L_{n}(\mathbb{C})$ ", Israel J. Math. 207:1 (2015), 435-448. MR 3358053 Zbl 06451119

[Kobayashi 2007] T. Kobayashi, "A generalized Cartan decomposition for the double coset space $\left(\mathrm{U}\left(n_{1}\right) \times \mathrm{U}\left(n_{2}\right) \times \mathrm{U}\left(n_{3}\right)\right) \backslash \mathrm{U}(n) /(\mathrm{U}(p) \times \mathrm{U}(q))$ ", J. Math. Soc. Japan 59:3 (2007), 669-691. MR 2008k:22017 Zbl 1124.22003

[Kostant 1978] B. Kostant, "On Whittaker vectors and representation theory”, Invent. Math. 48:2 (1978), 101-184. MR 80b:22020 Zbl 0405.22013

[Lapid and Mao 2014] E. Lapid and Z. Mao, "On a new functional equation for local integrals", pp. 261-294 in Automorphic forms and related geometry: assessing the legacy of I. I. PiatetskiShapiro, edited by J. W. Cogdell et al., Contemp. Math. 614, Amer. Math. Soc., Providence, RI, 2014. MR 3220931 Zbl 1306.22008

[Lapid and Rogawski 2003] E. M. Lapid and J. D. Rogawski, "Periods of Eisenstein series: the Galois case”, Duke Math. J. 120:1 (2003), 153-226. MR 2004m:11077 Zbl 1037.11033

[Offen 2011] O. Offen, "On local root numbers and distinction", J. Reine Angew. Math. 652 (2011), 165-205. MR 2012c:22025 Zbl 1278.22006

[Ok 1997] Y. Ok, Distinction and gamma factors at 1/2: supercuspidal case, Ph.D. thesis, Columbia University, 1997. MR 2716711

[Panichi 2001] M. N. Panichi, Charactérisations du spectre tempéré de $G L_{n}(\mathbb{C}) / G L_{n}(\mathbb{R})$, Ph.D. thesis, Paris 7 University, 2001.

[Serre 2002] J.-P. Serre, Galois cohomology, 2nd ed., Springer, Berlin, 2002. MR 2002i:12004 Zbl 1004.12003

[Springer 1984] T. A. Springer, Some results on algebraic groups with involutions, Publications mathématiques de l'Université Pierre et Marie Curie 61, Mathematisch Institut der Rÿksuniversiteit, 1984.

[Tadić 1985] M. Tadić, "Unitary representations of general linear group over real and complex field", preprint 22, Max Planck Institut für Mathematik, 1985, available at https://www.mpimbonn.mpg.de/preblob/5395.

[Vogan 1978] D. A. Vogan, Jr., "Gelfand-Kirillov dimension for Harish-Chandra modules", Invent. Math. 48:1 (1978), 75-98. MR 58 \#22205 Zbl 0389.17002

[Wallach 1988] N. R. Wallach, Real reductive groups, I, Pure and Applied Mathematics 132, Academic Press, Boston, 1988. MR 89i:22029 Zbl 0666.22002

[Wallach 1992] N. R. Wallach, Real reductive groups, II, Pure and Applied Mathematics 132, Academic Press, Boston, 1992. MR 93m:22018 Zbl 0785.22001 
Received October 12, 2014. Revised March 12, 2015.

ALEXANDER KEMARSKY

MATHEMATICS DEPARTMENT

TECHNION - ISRAEL INSTITUTE OF TECHNOLOGY

32000 HAIFA

ISRAEL

alexkem@tx.technion.ac.il 


\title{
PACIFIC JOURNAL OF MATHEMATICS
}

\author{
msp.org/pjm
}

Founded in 1951 by E. F. Beckenbach (1906-1982) and F. Wolf (1904-1989)

\section{EDITORS}

Don Blasius (Managing Editor)

Department of Mathematics

University of California

Los Angeles, CA 90095-1555

blasius@math.ucla.edu

\author{
Paul Balmer \\ Department of Mathematics \\ University of California \\ Los Angeles, CA 90095-1555 \\ balmer@math.ucla.edu \\ Robert Finn \\ Department of Mathematics \\ Stanford University \\ Stanford, CA 94305-2125 \\ finn@math.stanford.edu \\ Sorin Popa \\ Department of Mathematics \\ University of California \\ Los Angeles, CA 90095-1555 \\ popa@math.ucla.edu
}

\author{
Vyjayanthi Chari \\ Department of Mathematics \\ University of California \\ Riverside, CA 92521-0135 \\ chari@math.ucr.edu \\ Kefeng Liu \\ Department of Mathematics \\ University of California \\ Los Angeles, CA 90095-1555 \\ liu@math.ucla.edu \\ Jie Qing \\ Department of Mathematics \\ University of California \\ Santa Cruz, CA 95064 \\ qing@ cats.ucsc.edu
}

\section{PRODUCTION}

Silvio Levy, Scientific Editor, production@msp.org

\section{SUPPORTING INSTITUTIONS}

ACADEMIA SINICA, TAIPEI

CALIFORNIA INST. OF TECHNOLOGY

INST. DE MATEMÁTICA PURA E APLICADA

KEIO UNIVERSITY

MATH. SCIENCES RESEARCH INSTITUTE

NEW MEXICO STATE UNIV.

OREGON STATE UNIV.

\author{
STANFORD UNIVERSITY \\ UNIV. OF BRITISH COLUMBIA \\ UNIV. OF CALIFORNIA, BERKELEY \\ UNIV. OF CALIFORNIA, DAVIS \\ UNIV. OF CALIFORNIA, LOS ANGELES \\ UNIV. OF CALIFORNIA, RIVERSIDE \\ UNIV. OF CALIFORNIA, SAN DIEGO \\ UNIV. OF CALIF., SANTA BARBARA
}

\author{
Daryl Cooper \\ Department of Mathematics \\ University of California \\ Santa Barbara, CA 93106-3080 \\ cooper@math.ucsb.edu \\ Jiang-Hua Lu \\ Department of Mathematics \\ The University of Hong Kong \\ Pokfulam Rd., Hong Kong \\ jhlu@maths.hku.hk \\ Paul Yang \\ Department of Mathematics \\ Princeton University \\ Princeton NJ 08544-1000 \\ yang@math.princeton.edu
}

These supporting institutions contribute to the cost of publication of this Journal, but they are not owners or publishers and have no responsibility for its contents or policies.

See inside back cover or msp.org/pjm for submission instructions.

The subscription price for 2015 is US \$420/year for the electronic version, and \$570/year for print and electronic.

Subscriptions, requests for back issues and changes of subscribers address should be sent to Pacific Journal of Mathematics, P.O. Box 4163, Berkeley, CA 94704-0163, U.S.A. The Pacific Journal of Mathematics is indexed by Mathematical Reviews, Zentralblatt MATH, PASCAL CNRS Index, Referativnyi Zhurnal, Current Mathematical Publications and Web of Knowledge (Science Citation Index).

The Pacific Journal of Mathematics (ISSN 0030-8730) at the University of California, c/o Department of Mathematics, 798 Evans Hall \#3840, Berkeley, CA 94720-3840, is published twelve times a year. Periodical rate postage paid at Berkeley, CA 94704, and additional mailing offices. POSTMASTER: send address changes to Pacific Journal of Mathematics, P.O. Box 4163, Berkeley, CA 94704-0163.

PJM peer review and production are managed by EditFLOW ${ }^{\circledR}$ from Mathematical Sciences Publishers.

\section{PUBLISHED BY}

\section{mathematical sciences publishers \\ nonprofit scientific publishing}

http://msp.org/

(C) 2015 Mathematical Sciences Publishers 


\section{PACIFIC JOURNAL OF MATHEMATICS}

Volume $278 \quad$ No. $1 \quad$ November 2015

Growth tight actions

Goulnara N. Arzhantseva, Christopher H. Cashen and JING TAO

A flag structure on a cusped hyperbolic 3-manifold

ELISHA FALBEL and RAFAEL SANTOS THEBALDI

A new upper bound for the Dirac operators on hypersurfaces

Nicolas GinouX, GeORGES Habib and Simon RaUlot

Games and elementary equivalence of $\mathrm{II}_{1}$-factors

ISAAC GOLDBRING and THOMAS SINCLAIR

Grossberg-Karshon twisted cubes and hesitant walk avoidance

MEGUMI HARADA and EUNJEONG LEE

Gamma factors of distinguished representations of $\mathrm{GL}_{n}(\mathbb{C})$

ALEXANDER KEMARSKY

The $W$-entropy formula for the Witten Laplacian on manifolds with 173 time dependent metrics and potentials

SONGZI LI and XIANG-DONG LI

A diagrammatic categorification of the affine $q$-Schur algebra $\hat{\boldsymbol{S}}(n, n) 201$ for $n \geq 3$

MARCO MACKAAY and ANNE-LAURE THIEL

Showing distinctness of surface links by taking 2-dimensional braids

INASA NAKAMURA

Correction to Modular $L$-values of cubic level 